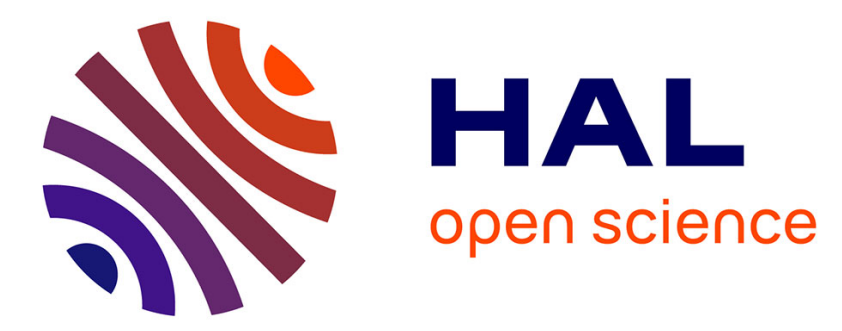

\title{
Hybrid Systems with State-Triggered Jumps: Sensitivity-Based Stability Analysis with Application to Trajectory Tracking
}

Mark Rijnen, Benjamin J.J.B. Biemond, Nathan van de Wouw, Alessandro Saccon, Henk Nijmeijer

\section{To cite this version:}

Mark Rijnen, Benjamin J.J.B. Biemond, Nathan van de Wouw, Alessandro Saccon, Henk Nijmeijer. Hybrid Systems with State-Triggered Jumps: Sensitivity-Based Stability Analysis with Application to Trajectory Tracking. 2018. hal-01727842v2

\section{HAL Id: hal-01727842 \\ https://hal.science/hal-01727842v2}

Preprint submitted on 4 Sep 2018

HAL is a multi-disciplinary open access archive for the deposit and dissemination of scientific research documents, whether they are published or not. The documents may come from teaching and research institutions in France or abroad, or from public or private research centers.
L'archive ouverte pluridisciplinaire HAL, est destinée au dépôt et à la diffusion de documents scientifiques de niveau recherche, publiés ou non, émanant des établissements d'enseignement et de recherche français ou étrangers, des laboratoires publics ou privés. 


\title{
Hybrid Systems with State-Triggered Jumps: Sensitivity-Based Stability Analysis with Application to Trajectory Tracking
}

\author{
Mark Rijnen, Benjamin Biemond, \\ Nathan van de Wouw, Alessandro Saccon, Henk Nijmeijer
}

\begin{abstract}
The definition of asymptotic stability for a trajectory of a hybrid system with state-triggered jumps is not straightforward. Nearby solutions jump at close but non-coincident times, making the standard notion of closeness, based on vector difference, unsuitable to compare trajectories point-wise in time. With tracking control as ultimate goal, we propose a notion of stability and a constructive stability proof based on sensitivity analysis applicable to single-jump-flow trajectories. A key role in the analysis is played by a time-triggered linear system, associated to the discontinuous trajectory of interest, whose uniform asymptotic stability suffices to guarantee the asymptotic stability of the original discontinuous trajectory. As an illustrative example, the stability analysis is applied to guarantee closed-loop stable tracking for a trajectory with velocity jumps of a 2 DoF mechanical system with unilateral constraint.
\end{abstract}

Index Terms-hybrid systems, stability, trajectory tracking, trajectories with jumps, sensitivity analysis

\section{INTRODUCTION}

$\mathbf{T}$ HIS paper studies the problem of defining and assessing local asymptotic stability of a trajectory of a hybrid dynamical system. These systems show both continuous (flow) and discrete (jump) dynamics [1]. Our analysis concerns, in particular, hybrid systems with (time- and) state-triggered jumps, where the state trajectory becomes discontinuous under the effect of the discrete dynamics. We will refer to this class of systems as hybrid systems with state-triggered jumps.

Hybrid systems with state-triggered jumps can be used to describe and analyze the behavior of mechanical systems with unilateral contact constraints employed in robotics and multibody dynamics [2]. Although other approaches worth considering for modeling and simulation of mechanical systems with unilateral constraints are available [3], [4], the hybrid system formalism [1] has been shown to be a viable modeling technique that allows for the description and control of, e.g., juggling and walking robots [5]-[7]. Whereas the stabilization of jumping trajectories of a mechanical system with unilateral constraints is the main motivation for our investigation, the approach and obtained results are applicable to the general

M.W.L.M. Rijnen, N. van de Wouw, A. Saccon, and H. Nijmeijer are with the Dept. of Mechanical Engineering, Eindhoven University of Technology, 5600MB Eindhoven, The Netherlands (e-mail: \{m.w.l.m.rijnen, n.v.d.wouw, a.saccon, h.nijmeijer $\} @$ tue.nl). N. van de Wouw is furthermore with the Dept. of Civil, Environmental and Geo-Engineering, University of Minnesota, MN 55455 Minneapolis, USA, and with the Delft Center for Systems and Control, Delft University of Technology, 2628CD Delft, The Netherlands.

J.J.B. Biemond is with the Netherlands Organization for Applied Scientific Research, TNO, Dept. of Optomechatronics, 2600AD Delft, The Netherlands class of hybrid systems and are therefore presented as such. More specifically, the proposed stability notion and sensitivitybased stability analysis concerns the specific type of trajectories termed single-jump-flow trajectories, characterized by continuous flow phases followed by single discrete jumps.

In earlier investigations [8]-[10], tracking problems for hybrid systems have been solved under the assumption that the jump times of the system and reference trajectory coincide. In that case, standard Lyapunov methods can be employed in terms of the classical Euclidean tracking error to perform stability analysis. The requirement that the jump times of the trajectories coincide with those of a reference trajectory is however stringent and this coincidence can generally not be assumed: this is not the case, in general, for hybrid systems with state-triggered jumps and, in particular, for hybrid systems that represent mechanical systems with unilateral constraints.

When reference and closed-loop jump times do not coincide, the Euclidean error between two trajectories shows a big increase whenever the system trajectory jumps and the reference trajectory does not or vice versa. This phenomenon is usually referred to as "peaking" [11]-[13]. A few approaches have been recently proposed in the literature to deal with the mismatch in the jump times by defining stability on the basis of a different notion of error/distance between singlejump-flow trajectories. In [14], the times belonging to the infinitesimal intervals around the jump times are neglected in defining the tracking problem. In [15], the reference trajectory together with a mirrored version of it has been used to construct a new error notion for the tracking problem of a ball in a polyhedral billiard. In [1, Section 5.3], the concept of graphical closeness of solutions is considered. In [13], [16], [17], the authors propose to simplify the stability analysis and tracking control design by suggesting to use a distance function between two trajectories that is invariant with respect to the discrete jump dynamics. In [18], the authors use gluing functions to perform a state-transformation turning the hybrid dynamics into (piecewise) continuous dynamics, removing the state jumps.

In this paper, we employ the notion of error introduced in [19] to define and analyze asymptotic stability of discontinuous trajectories and to propose a possible solution to the problem of tracking a reference trajectory for hybrid systems with state-triggered jumps. The employed error notion is based on extending the (reference) trajectory about the jump times and considering the distance between the state of a trajectory 
and that at the particular segment of the extended (reference) trajectory that has encountered the same number of jumps. This error will not, in particular, show any peaking and presents the basis for an effective trajectory tracking control approach named reference spreading control [19]-[21].

For smooth nonlinear control systems, the open- and closed-loop local stability of a continuous reference trajectory can be assessed via its associated time-varying linearization. The key contribution of this paper is to show that, for hybrid systems with state-triggered jumps, the local stability of a discontinuous single-jump-flow trajectory can be assessed via the study of its time-triggered linearization, a linear time-triggered hybrid system that emerges from the sensitivity analysis and that is independent of the reference extensions. The reference trajectory is assumed to satisfy a set of assumptions (in particular, transversality and absence of Zeno behavior) ensuring continuous dependence of impact times (and the solution away from impact times) with respect to variations of initial conditions and control inputs.

This paper is organized as follows. In Section II, hybrid systems with state-triggered jumps are reviewed and the problem definition is precisely stated together with key regularity assumptions. Section III reviews the concept of extended reference trajectory, that leads to the error notion used to define stability of discontinuous trajectories. This section also presents the main result of this paper: the ability to infer stability of a discontinuous trajectory of a hybrid system with statetriggered jumps by analysis of the stability of an associated time-triggered linear system (the hybrid linearization). Section IV applies the obtained results to a mechanical system with a unilateral constraint. Conclusions are presented in Section V.

\section{PRELIMINARIES AND PROBLEM STATEMENT}

\section{A. Hybrid systems}

A hybrid dynamical system can be represented schematically as in Fig. 1a. The system has a state $x \in \mathbb{R}^{n}$ that continuously evolves according to a control vector field $f: \mathbb{R}^{n} \times \mathbb{R}^{m} \rightarrow \mathbb{R}^{n}$, parameterized by the external input $u \in \mathbb{R}^{m}$. Continuous evolution is only possible when the state $x$ at a given time is in a closed set $C \subseteq \mathbb{R}^{n}$ called the flow set. Explicitly, the state evolution satisfies the differential equation

$$
\dot{x}=f(x, u), \quad x \in C .
$$

A jump in the state can occur whenever the state reaches a set $D \subseteq \mathbb{R}^{n}$, called the jump set. A jump implies an instantaneous state change according to the jump map $g: \mathbb{R}^{n} \rightarrow \mathbb{R}^{n}$.

In this paper, we consider unique solutions of hybrid systems. Aside from some basic regularity assumptions on the flow map $f$, uniqueness requires that whenever a jump occurs $(x \in D)$ evolving in $C$ is also no longer possible [1, Proposition 2.11]. A way to enforce this is to assume that $D \subseteq \partial C$, with $\partial C$ denoting the boundary of $C$, together with some transversality assumptions on the continuous flow to avoid that the flow is tangent to $D$ when it reaches it (grazing). Transversality (cf. [22]) plays an important role in this paper and will be discussed in more detail in the problem formulation in Section II-B.
We largely adopt the hybrid system notation from [1]. In particular, we employ the notion of hybrid time, which merges regular time $t \in \mathbb{R}$ with discrete time $j \in \mathbb{N}$. Discrete time should be thought of as a jump counter, indicating how many times the state has jumped, so that the state jumps satisfy

$$
x(t, j+1)=g(x(t, j)) \quad x \in D .
$$

All these notions are standard and we refer to [1, Definition 2.6] for the definition of a solution to (1a), (1b).

To allow for time-varying vector fields, jump maps, and time-varying flow and jump sets, the hybrid dynamical systems that we consider in this paper are (with slight abuse of notation) written as follows:

$$
\begin{aligned}
\dot{x} & =f(x, u, t, j), & & x \in C(t, j), \\
x^{+} & =g\left(x^{-}, t, j\right), & & x \in D(t, j),
\end{aligned}
$$

where $x^{+}:=x(t, j+1)$ and $x^{-}:=x(t, j)$. We refer to (2) as a Nonlinear State-Triggered Hybrid System (NSTHS) and represent it as shown in Figure $1 \mathrm{~b}$.

Remark 1. In (2), one could define a new state $(x, t, j)$ showing that (2) is just a special case of (1). However, we found that keeping the hybrid time $(t, j)$ explicit leads to a more intuitive understanding of the stability analysis and reference spreading control. In Section III, we show that the control law is of the form $u=u(x, t, j)$, depending therefore explicitly on the continuous flow state $x$ and hybrid time $(t, j)$.

Another reason to keep $(t, j)$ explicitly in (2) is that otherwise the proposed definition of discontinuous trajectory stability would require to treat $(t, j)$ differently than the other part of the state (otherwise, when time would be included in the state, a state perturbation would also perturb time).

In Section II-B and Appendix B, we will use the sets

$$
\begin{aligned}
C_{j} & :=\bigcup_{t \in \mathbb{R}} C(t, j) \times\{t\}, \\
D_{j} & :=\bigcup_{t \in \mathbb{R}} D(t, j) \times\{t\},
\end{aligned}
$$

to ease the derivations of the results. Note that $C_{j}$ and $D_{j} \subseteq \mathbb{R}^{n+1}$. For a given $j \in \mathbb{N}, C(t, j)$ and $D(t, j)$ can be interpreted as the "slices" of $C_{j}$ and $D_{j}$ at time $t$.

We adopt basic regularity assumptions for (2). To be precise, the flow map $f: \mathbb{R}^{n} \times \mathbb{R}^{m} \times \mathbb{R} \times \mathbb{N} \rightarrow \mathbb{R}^{n}$ is assumed to be locally Lipschitz with respect to the state $x$ on the set $\mathbb{R}^{n}$ and

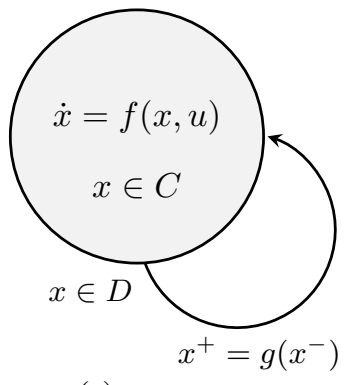

(a)

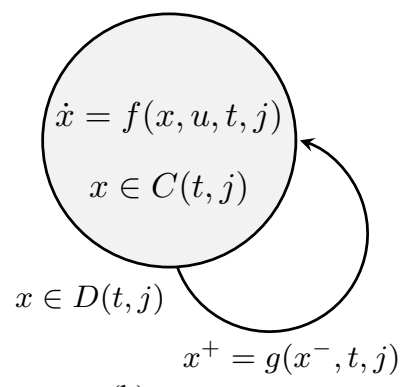

(b)
Fig. 1. Hybrid system with one mode of execution without (a) and with (b) explicit dependency on hybrid time $(t, j)$. 
input $u$ in $\mathbb{R}^{m}$, continuous and bounded in $t$ for each $x$ and $u$ and every fixed $j$. The jump map $g: \mathbb{R}^{n} \times \mathbb{R} \times \mathbb{N} \rightarrow \mathbb{R}^{n}$ is assumed to be continuous with respect to $x$ and $t$.

For the NSTHS (2), one may aim at designing a control law to achieve tracking of a given reference trajectory. A generic time-varying state feedback to achieve this goal is

$$
u=\kappa(x, t, j) \text {. }
$$

The closed-loop NSTHS (cl-NSTHS) resulting from substituting (5) into (2) is given by

$$
\begin{aligned}
\dot{x} & =f_{c l}(x, t, j) & & x \in C(t, j) \\
x^{+} & =g\left(x^{-}, t, j\right) & & x \in D(t, j),
\end{aligned}
$$

where $f_{c l}(x, t, j):=f(x, \kappa(x, t, j), t, j)$.

Suppose $x(t, j)$ is the solution to (6) for a given initial condition $x\left(t_{0}, 0\right)=x_{0} \in \operatorname{int} C\left(t_{0}, 0\right)$ (int means interior). The hybrid domain of $x(t, j)$ is written as (cf. [1, Definition 2.3])

$$
\operatorname{dom} x=\bigcup_{j=0}^{J_{x}-1} I_{x}^{j} \times\{j\}, \quad J_{x} \in \mathbb{N} \cup\{\infty\},
$$

with $I_{x}^{j}$ the closed (continuous-time) interval between the $j$-th and $(j+1)$-th jump events and $J_{x}$ the number of time intervals $\left(J_{x}=\infty\right.$, when an infinite number of jumps occur). The first interval $I_{x}^{0}$ starts at $t_{0}$. If $J_{x}<\infty$, the last interval $I_{x}^{J_{x}-1}$ ends at $t_{f} \in \mathbb{R} \cup\{\infty\}$. The $j$-th jump time is denoted $t_{j}$ so that

$$
I_{x}^{j}=\left[t_{j}, t_{j+1}\right]
$$

and also

$$
t_{j}=\min I_{x}^{j}
$$

The set of jump times associated to $x(t, j)$ is denoted

$$
E_{x}:=\bigcup_{j=1}^{J_{x}-1}\left\{t_{j}\right\} \times\{j-1\} .
$$

We conclude this section by introducing several notational conventions used to indicate the flow map. We will use $\mathcal{H}$ to indicate the closed-loop hybrid system defined by the quadruple $\left(f_{c l}, g, C, D\right)$, representing (6). Sometimes, we regard the solution to $\mathcal{H}$ starting from $x_{0}$ at hybrid time $\left(t_{0}, 0\right)$, just as a function of continuous time $t$ instead of hybrid time $(t, j) \geq\left(t_{0}, 0\right)$. To this end, with a slight abuse of notation, we will write $x(t)$ or, more explicitly, $\phi_{\mathcal{H}}\left(t, t_{0}, x_{0}\right)$ to indicate

$$
x(t)=\phi_{\mathcal{H}}\left(t, t_{0}, x_{0}\right):=x\left(t, j_{\mathcal{H}}\left(t, t_{0}, x_{0}\right)\right),
$$

with $x(t, j)$ as in (6) for $x\left(t_{0}, 0\right)=x_{0}$ and where $j_{\mathcal{H}}\left(t, t_{0}, x_{0}\right):=\max \{j \mid(t, j) \in \operatorname{dom} x(\cdot, \cdot)\}$ indicates the discrete time corresponding to assuming that, at time $t$, all discrete-time transitions have already occurred. We will also employ

$$
\varphi_{j}(t, s, x)
$$

to denote the flow (with no jumps) of the time-varying vector field $f_{c l}(\cdot, \cdot, j)$ in $(6 a)$ in the time interval $[s, t]$ with initial condition $x(s)=x$. Employing (11) and (12), and assuming to have just one jump at a time (this will be stated more formally in Assumption 1), one can write with no ambiguity $x(t, j)=\varphi_{j}\left(t, t_{j}, x\left(t_{j}, j\right)\right)=\varphi_{j}\left(t, t_{j}, \phi_{\mathcal{H}}\left(t_{j}, t_{0}, x_{0}\right)\right)$, as $j_{\mathcal{H}}\left(t_{j}, t_{0}, x_{0}\right)=j$ by definition of $t_{j}$. Furthermore, it holds that $x\left(t_{j}, j\right)=\phi_{\mathcal{H}}\left(t_{j}, t_{0}, x_{0}\right)=g\left(x\left(t_{j}, j-1\right), t_{j}, j-1\right)$.

\section{B. Problem formulation}

Consider a reference trajectory with jumps and denote it

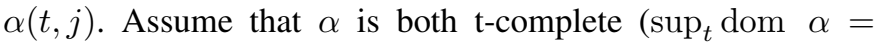
$\infty)$ and the unique solution to (2) with $\alpha\left(t_{0}, 0\right)=\alpha_{0} \in$ int $C\left(t_{0}, 0\right)$ and $u=\mu(t, j)$, continuous in $t$ for each $j$. The $j$-th event time of $\alpha$ is denoted $\tau_{j}$ and $I_{\alpha}^{j}=\left[\tau_{j}, \tau_{j+1}\right]$ is the $j$-th time interval between two consecutive events $\left(\tau_{0}=t_{0}\right)$. Denote $J_{\alpha}$ the number of intervals and $E_{\alpha}$ the set of event times. We consider the problem of assessing the stability of $\alpha(t, j)$, both in open- and closed-loop. Our stability analysis applies, in particular, to a single-jump-flow reference trajectory that is t-complete, non-Zeno, has a bounded inter-jump time, and intersects the jump set transversally. Furthermore, some minimal and easily encountered regularity conditions are also required, leading in total to six assumptions, detailed below. These assumptions are for example already satisfied for the simulation examples in [11], [13]-[16], [18], [20], [23]-[25]. We suggest the reader to skip the definition of the assumptions and explanatory remarks at first read, returning to them when necessary (in particular, when willing to understand the details of the proof of our main result in Section III-D).

Assumption 1 (t-complete, non-Zeno, bounded inter-jump time). The reference $\alpha$ is defined $\forall t>t_{0}$ ( $t$-complete) and $\exists \underline{\delta}_{t}>0$ such that $\tau_{j+1}-\tau_{j} \geq \underline{\delta}_{t}, \forall j \in\left\{0,1, \ldots, J_{\alpha}-1\right\}$ (non-Zeno). If $J_{\alpha}=\infty$, then moreover $\exists \bar{\delta}_{t}>0$ such that $\tau_{j+1}-\tau_{j} \leq \bar{\delta}_{t}, \forall j \in\left\{0,1, \ldots, J_{\alpha}-1\right\}$ (bounded inter-jump time).

As $\alpha$ is non-Zeno, $J_{\alpha}$ can become infinite only for $t \rightarrow \infty$. Completeness implies that $\alpha \in C\left(t, j_{\mathcal{H}}\left(t, t_{0}, \alpha_{0}\right)\right), \forall t \geq t_{0}$. We require the jumps to be transversal to the boundary of $C$. In (non-smooth) mechanics, for example, a jump is transversal when the impact between two convex bodies occurs with nonzero relative normal velocity (otherwise, a grazing impact occurs). To this end, we make use of guard functions $\gamma_{\alpha}$ that need to be defined only in a ball about each reference event.

Assumption 2 (Existence of a guard function). Given Assumption $1, \exists \varepsilon_{\gamma}>0$ and $c_{1}>0$, and a real-valued guard function $\gamma_{\alpha}(x, t, j), C^{1}$ with respect to both $x$ and $t$, $\forall j \in\left\{0,1, \ldots, J_{\alpha}-1\right\}$, such that

$$
\begin{array}{rlrl}
\gamma_{\alpha}(x, t, j) & >0 & & (x, t) \in B_{j} \cap \operatorname{int}\left(C_{j}\right) \\
\gamma_{\alpha}(x, t, j)=0 & (x, t) \in B_{j} \cap \partial C_{j}=: Z_{j} \\
\gamma_{\alpha}(x, t, j)<0 & (x, t) \in B_{j} \cap\left(\left(\mathbb{R}^{n} \times \mathbb{R}\right) \backslash C_{j}\right)
\end{array}
$$

where $B_{j}:=B_{\varepsilon_{\gamma}}\left(\alpha\left(\tau_{j+1}, j\right), \tau_{j+1}\right)$. In case $J_{\alpha}$ is finite, we pose $B_{J_{\alpha}-1}=\emptyset$. In (13), int and $\partial$ denote, respectively, the set's interior and boundary. We require $\gamma_{\alpha}(\cdot, \cdot, j)=0$ to define a co-dimension 1 manifold $\left(\gamma_{\alpha}\right.$ is the first coordinate of a $C^{1}$ diffeomorphism between $B_{\varepsilon_{\gamma}}$ and an open neighborhood of the origin on $\mathbb{R}^{n+1}$ ). We assume that

$$
Z_{j} \subset D_{j}
$$


and also

$$
\left\|\mathbf{D}_{1} \gamma_{\alpha}(\alpha(t, j), t, j)\right\| \leq c_{1},
$$

uniformly $\forall(t, j)=\left(\tau_{j+1}, j\right) \in E_{\alpha}$. In (15), $\mathbf{D}_{1}$ denotes partial differentiation with respect to the first argument.

In the assumption above, $B_{\varepsilon_{\gamma}}(x, t)$ denotes an open ball of radius $\varepsilon_{\gamma}$ about $(x, t)$, that is $B_{\varepsilon_{\gamma}}(x, t):=\left\{(y, s) \in \mathbb{R}^{n} \times\right.$ $\left.\mathbb{R} \mid\|(y, s)-(x, t)\|<\varepsilon_{\gamma}\right\}$.

Let us now formalize the assumption on the transversality property of the jumps of $\alpha$.

Assumption 3 (Transversality). Let Assumption 2 hold, implying the existence of $\gamma_{\alpha}$. There exist $c_{2}>0$ such that

$$
\begin{aligned}
& \mathbf{D}_{1} \gamma_{\alpha}(\alpha(t, j), t, j) \cdot f(\alpha(t, j), \mu(t, j), t, j)+ \\
& \mathbf{D}_{2} \gamma_{\alpha}(\alpha(t, j), t, j) \cdot 1 \leq-c_{2}
\end{aligned}
$$

for every event time $(t, j)=\left(\tau_{j+1}, j\right) \in E_{\alpha}$.

Aside from the assumptions on $\alpha$, we also pose continuity conditions on the jump map $g$ and vector field $f$.

Assumption 4 (Locally differentiable jump map). Given Assumptions 1 and 2 , we require $g(\cdot, \cdot, j)$ to be $C^{1}$ in the open ball $B_{\varepsilon_{\gamma}}\left(\alpha\left(\tau_{j+1}, j\right), \tau_{j+1}\right), \forall j \in\left\{0,1, \ldots, J_{\alpha}-1\right\}$.

Assumption 5 (Uniform Lipschitz condition on $f$ ). In a neighborhood of the reference state-input trajectory $(\alpha, \mu)$, $f(x, u, t, j)$ is Lipschitz with respect to $x$ and $u$, uniformly in $t$ and $j$. Namely, we assume that $\exists \varepsilon_{L}>0$ independent of $(t, j)$ and $\exists L$ for which, $\forall j \in\left\{0,1, \ldots, J_{\alpha}-1\right\}, \| f(x, u, t, j)-$ $f(y, v, t, j) \|<L(\|x-y\|+\|u-v\|), \forall t \in\left(\tau_{j}-\varepsilon_{L}, \tau_{j+1}+\varepsilon_{L}\right)$, $x, y \in B_{\varepsilon_{L}}(\bar{\alpha}(t, j))$, and $u, v \in B_{\varepsilon_{L}}(\bar{\mu}(t, j))$.

Remark 2. The Lipschitz constant $L$ is defined for time intervals $t \in\left(\tau_{j}-\varepsilon_{L}, \tau_{j+1}+\varepsilon_{L}\right)$ that are not contained in dom $\alpha$. Therefore, an extended reference state-input trajectory $(\bar{\alpha}, \bar{\mu})$, for which dom $\alpha \subset \operatorname{dom} \bar{\alpha}$, is used in its definition. See Section III-A for further details.

The following assumption, imposing natural conditions on $\alpha$ and the state-triggered hybrid system, ensures that trajectories sufficiently close to $\alpha(t, j)$ are also t-complete. In the assumption, $\mathcal{T}_{p} S$ denotes the tangent cone to $S$ at $p$ as defined, e.g., in [1, Definition 5.12].

Assumption 6 (Local existence of t-complete solutions). Let Assumptions 1 to 3 hold. Every state-time pair $(x, t) \in Z_{j}$ of the reference event $\left(\alpha\left(\tau_{j+1}, j\right), \tau_{j+1}\right)$, with $Z_{j}$ defined as in (13) and $j \in\left\{0,1, \ldots, J_{\alpha}-1\right\}$, is mapped by the jump map $g$ to the subsequent flow set, while avoiding the jump set, i.e.,

$$
(x, t) \in Z_{j} \Longrightarrow(g(x, t, j), t) \in C_{j+1} \backslash D_{j+1} .
$$

Furthermore, we assume that $f(x, u, t, j)$ satisfies

$$
(f(x, u, t, j), 1) \in \mathcal{T}_{(x, t)} C_{j},
$$

$\forall j \in\left\{0,1, \ldots, J_{\alpha}-1\right\}, \forall u$ in a uniform neighborhood of $\bar{\mu}(t, j)$, and for $(x, t) \in \partial C_{j} \cap\left(U_{j} \backslash B_{j}\right)$, with $U_{j}$ defined below and $B_{j}$ as in (13). In (17),

$$
U_{j}:=\bigcup_{t \in\left[\tau_{j}-\varepsilon_{C}, \tau_{j+1}\right]}\left(B_{\varepsilon_{C}}(\bar{\alpha}(t, j)) \times\{t\}\right),
$$

that is $U_{j}$ denotes the set of all state-time pairs $(x, t)$ contained in the tube of size $\varepsilon_{C}>0$ about the extended reference trajectory $\bar{\alpha}$.

Remark 3. The assumption allows to handle solutions close to the reference trajectory that are only defined on a finite time domain, without resorting to advanced concepts such as preasymptotic stability [1, Chapter 7]. The required properties guarantee that, away from the jump event times $\left(\tau_{j+1}, j\right)$ (hence the asymmetry in the definition of $U_{j}$ ), trajectories in a neighborhood of $\alpha(t, j)$ remain in the flow set and that, after each jump, flowing is always possible (for the points that are the image through the impact map of the pre-jump states, the vector field is directed inward the flow set). To account for any differences in jump time of the nearby solutions, the given properties are required to hold on a larger time domain than dom $\alpha$, requiring (similarly to what is discussed for the previous assumption) the availability of an extended reference trajectory $\bar{\alpha}$ (see Section III-A).

In the next section, we introduce the concept of reference spreading error between two trajectories and we proceed with the definition of time-triggered linearization of the NSTHS (2) about a reference trajectory $\alpha$. Furthemore, we show that the stability of this time-triggered linear hybrid system (about the origin) implies local stability of the NSTHS about $\alpha$.

\section{SENSITIVITY-BASED STABILITY ANALYSIS OF JUMPING TRAJECTORIES}

In this section, the stability properties of the reference trajectory $\alpha(t, j)$ of the cl-NSTHS in (6) are analyzed. First, the notion of extended (reference) trajectory will be introduced to define a useful error measure on the basis of which a definition of stability of the jumping reference trajectory will be given. Secondly, a time-triggered linear hybrid system will be defined, the trajectories of which can be used to approximate those of the NSTHS starting near the reference trajectory. This section is concluded by showing that a switching controller can be designed using this linear hybrid system, as asymptotic stability of this system in closed-loop implies asymptotic stability of the reference trajectory of the cl-NSTHS in (6).

\section{A. Error definition}

Any difference between the initial conditions $x_{0}$ and $\alpha_{0}$ will most likely result in differences between the jump times of the reference and those of the closed-loop system. Therefore, as mentioned in the introduction, designing a tracking controller based on the Euclidean error defined as the difference between the current state $x$ and $\alpha$ (for the same $t$ only) may easily result in poor tracking performance (see [15], [20] for simulation examples supporting this statement). Therefore, as suggested in [19], for every value of the jump counter $j$, the segment of the reference trajectory corresponding to the interval $I_{\alpha}^{j} \times\{j\}$ is extended using the vector field (2a) with input $u=\bar{\mu}(t, j)$ that is the continuously extended version (by design) of $\mu(t, j)$ such that each reference segment is defined for all $t \in\left[t_{0}, t_{f}\right]$. This extended reference trajectory is denoted $\bar{\alpha}$ and is thus defined for all $(t, j) \in\left[t_{0}, t_{f}\right] \times\left\{0,1, \ldots, J_{\alpha}-1\right\}=: \bar{I}_{\alpha}$. 
Note that $(\bar{\alpha}, \bar{\mu})$ coincides with $(\alpha, \mu)$ for $(t, j) \in \operatorname{dom} \alpha$. Formally, $\forall j \in\left\{0,1, \ldots, J_{\alpha}-1\right\}$, we define $t \mapsto \bar{\alpha}(t, j)$, $t \in\left[t_{0}, t_{f}\right]$, as the solution to

$$
\dot{\bar{\alpha}}=f(\bar{\alpha}, \bar{\mu}(t, j), t, j), \quad(t, j) \in \bar{I}_{\alpha},
$$

for $\bar{\alpha}\left(\tau_{j}, j\right)=\alpha\left(\tau_{j}, j\right)$, where $\tau_{j}$ denotes the $j$-th jump time $\left(\tau_{0}=t_{0}\right)$. The construction of the extended trajectory requires both forward and backward integration of the vector field to extend the $j$-th reference segment outside the original interval $\left[\tau_{j}, \tau_{j+1}\right]$.

Remark 4. Tracking the reference trajectory $\alpha$ using the notion of extended trajectories as proposed in [19] requires more than just the state of the reference at the current time, it requires knowledge of the future reference. The trajectory $\alpha$ needs to be known beforehand or, at least, the segment up to the next jump of $\alpha$. If the closed-loop system encounters a jump prior to the reference trajectory, for example, it already needs to know what the desired motion is after the jump event. This knowledge comes from the backwards integration of the vector field from the time where the jump is expected to occur (for incremented jump counter), i.e. the reference jump time.

Note that when a hybrid trajectory that is a solution to (2) is projected onto the continuous time domain, it is single valued for all $t$ in its domain except for the jump times. This is not the case for $\bar{\alpha}$, as now for each time $t$ there are $J_{\alpha}$ extended "reference" trajectories discriminated by the counter $j$. This counter will be used to compare the state $x$ at any given time to the relevant branch of the extended reference $\bar{\alpha}$. Note furthermore that the domain $\bar{I}_{\alpha}$ is not a hybrid time domain as in [1], because the natural ordering of its points is lost, i.e. the boundaries of the time intervals do not form an increasing sequence. The construction of the extended trajectory $\bar{\alpha}(t, j)$ requires the vector field $f$ in (2a), with $u=\bar{\mu}(t, j)$, to be defined (by design) outside the domain $C(t, j)$, ignoring the presence of the jump set and allowing the time integration to continue beyond it (see [20], [21] for examples). The extended reference trajectory $\bar{\alpha}$ allows to define the tracking error as

$$
e(t, j):=x(t, j)-\bar{\alpha}(t, j) \quad(t, j) \in \operatorname{dom} x .
$$

In a small neighborhood of dom $\alpha$, this definition of error is similar to the concept of graphical closeness of graphs of solutions as described in [1, Definition 4.11]. A graphical representation of $\bar{\alpha}$, its "extended" hybrid time domain $\bar{I}_{\alpha}$, and the tracking error $e(t, j)$ is given in Fig. 2.

\section{B. Stability definition}

We provide here the definition of stability and asymptotic stability for a single-jump-flow trajectory $\alpha$ of the NSTHS (6), using the notion of error introduced in the previous section.

Definition 1 (Stability). Given $t_{0}$, a trajectory $\alpha$ of (6) that is t-complete is said to be stable if for all $\varepsilon>0$ there exists a $\delta>0$ such that for every trajectory $x$ of (6) satisfying $\left\|x\left(t_{0}, 0\right)-\alpha\left(t_{0}, 0\right)\right\|<\delta$, it holds that a) $J_{x}=J_{\alpha}$, b) for all $(t, j) \in \operatorname{dom} x,\|x(t, j)-\bar{\alpha}(t, j)\|<\varepsilon$ and, $c)$ for all $j \in\left\{1, \ldots, J_{x}-1\right\},\left|t_{j}-\tau_{j}\right|<\varepsilon$, where $\bar{\alpha}$ is the extension of $\alpha$ defined in (19) and $J_{x}$ and $J_{\alpha}$ are, respectively, the number
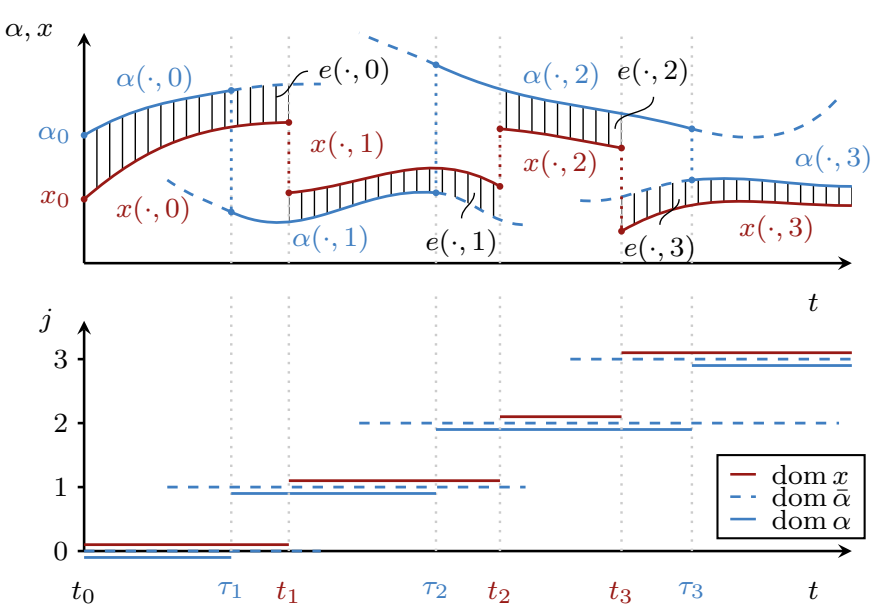

Fig. 2. The extended reference $\bar{\alpha}$, the tracking error $e$ for a trajectory $x$, and their corresponding time domains.

of time intervals $I_{x}^{j}$ and $I_{\alpha}^{j}$ defined as in (8) for the trajectories $x(t, j)$ and $\alpha(t, j)$, possibly infinite.

Remark 5. This definition of stability makes use of the error definition in (20), which is the Euclidean distance between the state $x$ and the extended reference trajectory $\bar{\alpha}$ for each $(t, j) \in \operatorname{dom} x$. Note that $e(t, j)$ has the same time domain as $x(t, j)$ and requires dom $x \subseteq \bar{I}_{\alpha}$ for it to be defined. The latter is guaranteed when $J_{x} \leq J_{\alpha}$ which is satisfied with strict equality in the case of stability of $\alpha$, for sufficiently small $\delta$ (as in Definition 1). The dependency of the jump times of $x(t, j)$ on initial condition $x_{0}$ will be elaborated on in Lemma $1 . \triangle$

Definition 2 (Attractivity). Given a trajectory $\alpha$ of (6) that is t-complete, we say that $\alpha$ is attractive if there exists a $\delta>0$ such that $\left\|x\left(t_{0}, 0\right)-\alpha\left(t_{0}, 0\right)\right\|<\delta$ implies, firstly, that $J_{x}=$ $J_{\alpha}$ and, secondly, that $\|x(t, j)-\bar{\alpha}(t, j)\| \rightarrow 0$ for $t \rightarrow \infty$ with $(t, j) \in \operatorname{dom} x$, where $\bar{\alpha}$ is the extension of $\alpha$ defined in (19) and $J_{x}$ and $J_{\alpha}$ are, respectively, the number of time intervals $I_{x}^{j}$ and $I_{\alpha}^{j}$ defined as in (8) for the trajectories $x(t, j)$ and $\alpha(t, j)$. If $J_{\alpha}=\infty$, we require that furthermore $\left|t_{j}-\tau_{j}\right| \rightarrow 0$ for $j \rightarrow \infty$.

Definition 3 (Asymptotic stability). A trajectory $\alpha$ of the $\mathrm{cl}$ NSTHS (6) is asymptotically stable if it is stable and attractive, respectively in the sense of Definitions 1 and 2.

\section{Linear time-triggered hybrid system (LTTHS)}

As mentioned in the previous section, a nonzero tracking error will likely result in a mismatch between the closed-loop jump times $t_{j}$ and the reference jump times $\tau_{j}$. The times $t_{j}$, with $j \in\left\{1,2, \ldots, J_{x}-1\right\}$ are not known in advance. Instead, as the reference $\alpha$ is assumed to be known, the event times $\tau_{j}$ are known. Next, we construct a linear time-triggered system, that jumps at the reference jump times $\tau_{j}$, and solutions of which can be used to approximate the error in the state evolution for (6) in a neighborhood of $\alpha$. We can use this timetriggered linear system to design a stabilizing feedback of the form (5) and we show that asymptotic stability of this closedloop linear hybrid system implies (local) asymptotic stability of the reference trajectory $\alpha$ for the original cl-NSTHS (6). 
We refer to this linear system with jumps at the times $\tau_{j}$ as the Linear Time-Triggered Hybrid System (LTTHS) associated to the reference trajectory $\alpha$. The key feature of this LTTHS is that it converts the state-triggered behavior of (2) to a time-triggered one (cf. [19]) and incorporates a first order approximation of the state-jumps (originally at slightly different times) in the definition of the jump map, see e.g. [19], [26]. We will show that asymptotic stability of $\alpha$ in the sense of Definition 3 can be assessed by studying the LTTHS corresponding to (2) and the state-input trajectory $(\alpha(t, j), \mu(t, j))$, with $(t, j) \in \operatorname{dom} \alpha$. Hence, the stability analysis is significantly simplified as $\alpha(t, j)$ and the LTTHS jump at the same time. Let us now formally define the LTTHS.

Definition 4 (LTTHS). The linear time-triggered hybrid system associated to trajectory $\alpha$ and NSTHS (2) is given by

$$
\begin{aligned}
\dot{z} & =A(t, j) z+B(t, j) v & & (t, j) \in \operatorname{dom} \alpha \\
z^{+} & =G(j) z^{-} & & (t, j) \in E_{\alpha}
\end{aligned}
$$

with initial condition $z\left(t_{0}, 0\right)=z_{0}$ and where $z^{+}:=z(t, j+$ $1), z^{-}:=z(t, j)$

$$
\begin{aligned}
& A(t, j):=\mathbf{D}_{1} f(\alpha(t, j), \mu(t, j), t, j), \\
& B(t, j):=\mathbf{D}_{2} f(\alpha(t, j), \mu(t, j), t, j),
\end{aligned}
$$

and

$$
G(j):=\frac{f^{+}-\dot{g}^{-}}{\dot{\gamma}_{\alpha}^{-}} \mathbf{D}_{1} \gamma_{\alpha}^{-}+\mathbf{D}_{1} g^{-}
$$

with

$$
\begin{aligned}
f^{+} & =f\left(\alpha^{+}, \mu^{+}, \tau, j+1\right) \\
f^{-} & =f\left(\alpha^{-}, \mu^{-}, \tau, j\right) \\
g^{-} & =g\left(\alpha^{-}, \tau, j\right) \\
\dot{g}^{-} & =\left(\mathbf{D}_{1} g^{-}\right) f^{-}+\mathbf{D}_{2} g^{-} \\
\gamma_{\alpha}^{-} & =\gamma_{\alpha}\left(\alpha^{-}, \tau, j\right) \\
\dot{\gamma}_{\alpha}^{-} & =\left(\mathbf{D}_{1} \gamma_{\alpha}^{-}\right) f^{-}+\mathbf{D}_{2} \gamma_{\alpha}^{-}
\end{aligned}
$$

where $\tau=\tau_{j+1}, \alpha^{+}=\alpha(\tau, j+1), \alpha^{-}=\alpha(\tau, j), \mu^{+}=$ $\mu(\tau, j+1), \mu^{-}=\mu(\tau, j)$, and $\gamma_{\alpha}(\cdot, \cdot, j)$ denotes the guard functions (see Section II-B).

As will be clarified later on, the linear hybrid system (21)(30) provides an approximation of the NSTHS in the sense that a trajectory of the NSTHS starting at a perturbed initial condition $x_{0}=\alpha_{0}+z_{0}$ with perturbed input $\mu(t, j)+v(t, j)$ can be approximated as $x(t, j)=\bar{\alpha}(t, j)+\bar{z}(t, j)+o\left(\left\|z_{0}\right\|\right)$ for $(t, j) \in \operatorname{dom} x$. In this, $\bar{z}$ is the extended trajectory of $z$ obtained in the same way as $\bar{\alpha}$, that is, for each $\mathrm{j}$, it follows from integrating the vector field $\dot{z}=A(t) z+B(t) v(t, j)$ with $t \in\left[t_{0}, t_{f}\right]$ forward and backward in time from initial condition $\bar{z}\left(\tau_{j}, j\right)=z\left(\tau_{j}, j\right)$. The term $o\left(\left\|z_{0}\right\|\right)$ denotes a perturbation that is of order higher than one. The state $\bar{z}$ is thus a first-order approximation of the error (20) if $J_{x} \leq J_{\alpha}$.

Given the time-triggered "linearization" of the NSTHS about a trajectory $\alpha$, one can attempt to design a control law to make the origin of the LTTHS uniformly asymptotically stable. The uniformity property will be required for showing that $\alpha$ is an asymptotically stable trajectory of the NSTHS if the origin of the linearization indeed satisfies the posed stability properties. This will be discussed in Section III-D. Considering the fact that the LTTHS is a "linearization", we restrict attention to feedbacks of the form

$$
v(t, j)=-K(t, j) z(t, j) .
$$

By suitably designing the time-varying feedback gain $K$, uniform asymptotic stability of the origin of the closed-loop LTTHS (cl-LTTHS) can be achieved, by which we mean the following (see [27, Section 3.1]):

Definition 5 (LTTHS: Uniform asymptotic stability). The origin of the cl-LTTHS (21)-(31) is uniformly asymptotically stable if for every $\varepsilon>0$ and $T_{0} \geq t_{0}$ there exists $a \delta$, independent of $T_{0}$, such that $\left|z\left(T_{0}, j_{T_{0}}\right)\right| \leq \delta$ implies $|z(t, j)| \leq \varepsilon$ for all $(t, j) \in \operatorname{dom} \alpha$ with $t \geq T_{0}$ and that $\lim _{t \rightarrow \infty}|z(t, j)|=0$. In this, $j_{T_{0}}$ is the counter $j$ corresponding to the time $T_{0}$, that is, the largest $j$ such that $T_{0} \geq \tau_{j}$.

The stability assessment of (21) in closed-loop (i.e. with feedback (31)) is well established in literature, see e.g. [27, Sections 3.2 and 6.4] and [28].

Using (31) and the fact that $\bar{z}(t, j)$ is a local approximation of the error (20), we obtain a cl-NSTHS with input

$$
u=\kappa(x, t, j)=\bar{\mu}(t, j)-\bar{K}(t, j)(x(t, j)-\bar{\alpha}(t, j)) .
$$

Note that where $z$ is defined for all $(t, j) \in \operatorname{dom} \alpha$, the state $x$ and error $e$ have a different hybrid time domain that is not known in advance. For the feedback law to be well-defined, we thus require the time-varying feedback gain to be defined for a larger time domain than dom $\alpha$, i.e. for all $(t, j) \in \bar{I}_{\alpha}=$ $\left[t_{0}, t_{f}\right] \times\left\{0,1, \ldots, J_{\alpha}-1\right\}$. Therefore, in (32), we introduced $\bar{K}(t, j)$ representing the feedback gain $K(t, j)$, but extended such that it is defined for all $(t, j) \in \bar{I}_{\alpha}$. Due to this extension, the feedback control is defined for all $(t, j) \in \operatorname{dom} x$ (as long as $J_{x} \leq J_{\alpha}$ ). Several approaches are possible in constructing these extensions. However, the extension of the feedback gains $\bar{K}(t, j)$ does not influence the LTTHS since it only depends on the perturbation input $v(t, j)$ for $(t, j) \in \operatorname{dom} \alpha$ (see [20]). This property is explained further in Appendix D.

\section{Main stability result}

The problem considered in this paper is that of assessing the stability properties of a jumping reference trajectory $\alpha(t, j)$ of the cl-NSTHS (6) under Assumptions 1 to 6. As a stepping stone, a key fact that we will exploit is that, for any finite time $T>t_{0}$, the jump times of the cl-NSTHS (6) in dom $x \cap\left[t_{0}, T\right] \times \mathbb{N}$ depend in a continuously differentiable fashion on the initial condition $x_{0}$ as long as $x_{0}$ is chosen in a sufficiently small neighborhood of $\alpha_{0}$ dependent on $T$. This result follows from Assumptions 1 to 4 and Assumption 6 as well as some minimal regularity assumptions on the vector field $f_{c l}$ and jump map $g$, and it is proved in Lemma 1. Note that the size of this neighborhood might vanish for $T \rightarrow \infty$. In order to conclude stability, this dependency of jump times on initial condition, Assumption 5, and stability of the linear error dynamics are key. Let us first consider the former property for which we define a jump counter function for the reference trajectory $\alpha$. 
Definition 6 (Jump counter function). For a given $T>t_{0}$ and hybrid trajectory $\alpha$, denote with $j_{\alpha}(T)$ the number of encountered jumps of the reference trajectory for $t \leq T$.

The jump counter function $j_{\alpha}:\left(\tau_{1}, \infty\right) \rightarrow \mathbb{N}$ is right continuous and satisfies the inequality $\tau_{j_{\alpha}(T)} \leq T$. It is equal to the jump counter $j_{\mathcal{H}}$, introduced in Section II, when evaluated along the specific trajectory $\alpha$, i.e. $j_{\alpha}(T)=j_{\mathcal{H}}\left(T, t_{0}, \alpha_{0}\right)$. The following lemma now holds.

Lemma 1. For the hybrid system (6), assume that the vector field $f_{c l}$ is locally Lipschitz with respect to $x$ and continuous and bounded in $t$. Let $\alpha$ denote a reference trajectory of the NSTHS (6) satisfying Assumptions 1, 2, 3, 4, and 6, with initial condition $\alpha\left(t_{0}, 0\right)=\alpha_{0} \in \operatorname{int}\left(C\left(t_{0}, 0\right)\right)$. As before, $\tau_{j}, j \in$ $\left\{0,1, \ldots, J_{\alpha}-1\right\}$, indicates the nominal event times.

There exists a function $\delta_{0}:\left(\tau_{1}, \infty\right) \rightarrow \mathbb{R}_{>0}$ such that, for any $T>\tau_{1}$, a trajectory of the cl-NSTHS (6) with initial condition $x\left(t_{0}, 0\right)=x_{0}$ satisfying

$$
\left\|x_{0}-\alpha_{0}\right\|<\delta_{0}(T) \quad \text { with } \quad B_{\delta_{0}(T)}\left(\alpha_{0}\right) \subset C\left(t_{0}, 0\right)
$$

is defined at least up to time $T$ and jumps at least $j_{\alpha}(T)-1$ times in the interval $t \in\left[t_{0}, T\right]$. Furthermore, when $j_{\alpha}(T) \geq$ 2 , the function $\delta_{0}(\cdot)$ can be chosen such that, in addition, every jump time $t_{j}$ except the last is bracketed by the nominal jump times $\tau_{j-1}$ and $\tau_{j+1}$, i.e.

$$
\tau_{j-1} \leq t_{j} \leq \tau_{j+1}, \quad \text { for } j \in\left\{1,2, \ldots, j_{\alpha}(T)-1\right\} .
$$

Proof. The proof of Lemma 1 is presented in Appendix A.

Stability of the reference trajectory $\alpha$ for the cl-NSTHS (6), (32) can now be related to the stability of the cl-LTTHS (21)(31), resulting in the main result of this work given below.

Theorem 1. Adopt Assumptions 1-6. Let a state-input trajectory $(\alpha, \mu)$ be a solution to the cl-NSTHS (6), (32). If the origin of the associated LTTHS (21)-(30) in closed-loop with control law (31) is uniformly asymptotically stable, then, $\alpha(t, j)$ is a (locally) asymptotically stable trajectory of the cl-NSTHS (6), (32) in the sense of Definition 3.

Proof. The proof of Theorem 1 is given in Appendix B.

To bridge the gap between this work and other approaches in literature for analyzing stability of state-triggered hybrid systems, in Appendix $\mathrm{C}$ we show that uniform asymptotic stability of the cl-LTTHS (21)-(31) also implies asymptotic stability of the reference trajectory $\alpha$ for (a variant of the) clNSTHS (6), (32) in terms of the distance function defined in [13], [16]. Mind that, in this implication, the hybrid system class that is considered is reduced to one where the jump map $g$, and the flow and jump sets $C$ and $D$, respectively, do not depend on time explicitly as the distance function in [13], [16] does not accommodate such time-varying nature.

\section{ILlustratiVE EXAMPLE}

In this section, a trajectory tracking example for mechanical systems with a unilateral constraint is presented. A circular billiard with periodic reference trajectory is considered. In this, the number of jumps $J_{\alpha}-1$ becomes infinite as $t \rightarrow \infty$. We

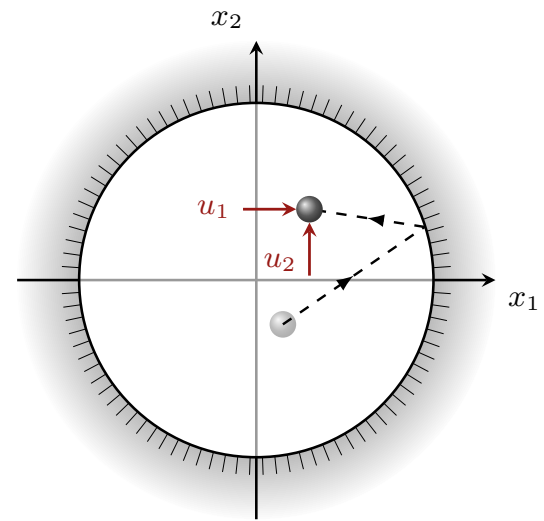

Fig. 3. Schematic representation of a circular billiard.

only consider a periodic reference here for simplicity, but for the theory presented in this work no periodicity is required.

Consider the time-invariant system depicted in Fig. 3 (that is also considered in [11] for fully elastic restitution) consisting of an actuated point mass moving in a plane that is confined by a circular boundary. Such a system is commonly referred to in literature as a billiard.

The position of the point mass in the plane, at a particular time, is given by the coordinates $x_{1}$ and $x_{2}$ (see Fig. 3 ). The velocity components of the mass in the $x_{1}$ and $x_{2}$ direction are denoted by $x_{3}=\dot{x}_{1}$ and $x_{4}=\dot{x}_{2}$, respectively. The state of the point mass thus is $x=\left[\begin{array}{llll}x_{1} & x_{2} & x_{3} & x_{4}\end{array}\right]^{T}$ and accelerations can be imposed in $x_{1}$ and $x_{2}$ direction (denoted $u_{1}$, respectively $u_{2}$, such that $u=\left[\begin{array}{ll}u_{1} & u_{2}\end{array}\right]^{T}$ ). A rigid object confines the space in which the mass can move such that the jump set is defined as $D=\left\{x \in \mathbb{R}^{4} \mid x_{1}^{2}+x_{2}^{2}=1, x_{1} x_{3}+x_{2} x_{4}>0\right\}$. The flow set therefore becomes $C=\left\{x \in \mathbb{R}^{4} \mid x_{1}^{2}+x_{2}^{2} \leq 1\right\}$ and a suitable guard function for the system is $\gamma_{\alpha}=1-x_{1}^{2}-x_{2}^{2}$ (satisfying Assumption 2). Whenever the mass impacts the boundary, partially elastic restitution occurs with a coefficient of restitution $e$. The system can be described by (2), with

$$
\begin{aligned}
& f(x, u, t, j)=\left[\begin{array}{llll}
x_{3} & x_{4} & u_{1} & u_{2}
\end{array}\right]^{T}=: A x+B u, \\
& A=\left[\begin{array}{cc}
0_{2 \times 2} & I_{2} \\
0_{2 \times 2} & 0_{2 \times 2}
\end{array}\right], \quad B=\left[\begin{array}{c}
0_{2 \times 2} \\
I_{2}
\end{array}\right],
\end{aligned}
$$

and

$$
g(x, t, j)=\left[\begin{array}{c}
x_{1} \\
x_{2} \\
\left(x_{2}^{2}-e x_{1}^{2}\right) x_{3}-(1+e) x_{1} x_{2} x_{4} \\
\left(x_{1}^{2}-e x_{2}^{2}\right) x_{4}-(1+e) x_{1} x_{2} x_{3}
\end{array}\right],
$$

cf. [11]. In this example, it is assumed that the interaction between actuated mass and obstacle can fully be modeled using the impact law, that is, periods of persistent unilateral contact do not occur and (finite) contact forces thus need not be included in the vector field $f$. Furthermore, the pair $(\alpha, \mu)$ is considered to be such that grazing incidence of the point mass on the obstacle is avoided (cf. Assumption 3). Note that, in this example, the flow set $C$, jump set $D$, vector field $f$, and jump map $g$ do not depend explicitly on the hybrid time $(t, j)$. This time-invariance is chosen here for the sake of simplicity 

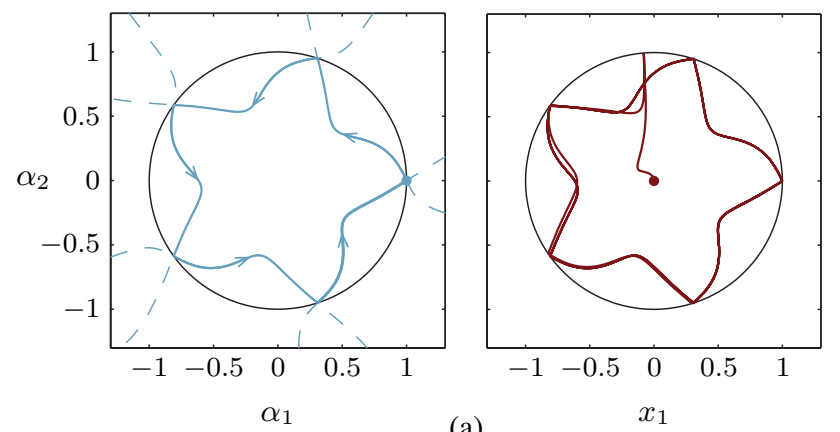

(a)
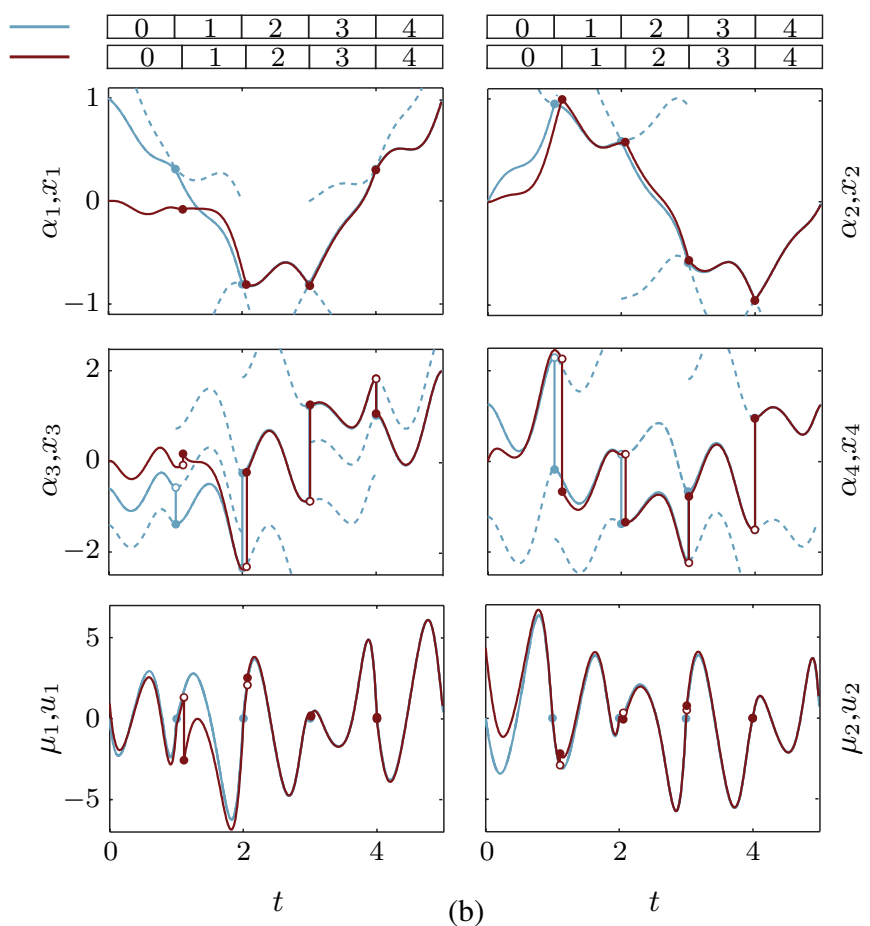

(b)

Fig. 4. The reference trajectory $\alpha(t, j)$ (light blue) with extensions (dashed) and a tracking solution $x(t, j)$ (dark red) to the cl-NSTHS in Fig. 3 in the $x_{1}, x_{2}$-plane (where the dot indicates the initial position) (a) and the evolution of state and input over time (where the open and closed dots indicate the left, respectively, the right limit) (b).

of the analysis only, and is not required for the applicability of the theory in this work.

Consider the reference trajectory shown in Fig. 4a. It is a periodic solution to the system description above where the state returns back to its initial condition after five impacts with coefficient of restitution $e=0.3$. The impacts are separated in time by a period $\tau=1$ (cf. Assumption 1) and since the trajectory starts at the boundary it follows that $\tau_{j}=j \tau$ for $j=0,1, \ldots$ (taking $t_{0}=0$ ). As can be discerned from the fact that the trajectory segments between impacts are curved, the input $\mu(t, j)$ is not zero for all time. As a consequence, constructing the extended trajectory $\bar{\alpha}$ as described in Section III will be different from the strategy of "mirroring" as is used for billiards in [15], [24], [25]. Furthermore, note that the coefficient of restitution is significantly below one. If the input would be zero and $e$ equal to one, for this particular system, the two approaches would result in the same behavior.
The strategy taken in this paper however is more generic and applicable to a larger class of systems. It allows to exploit knowledge of the complete reference trajectory, therewith making optimal control approaches within reach.

It is straightforward to show that for the specific example Assumptions 1-6 are satisfied. Due to space restrictions, this proof is not included here but it is available upon request.

To design the stabilizing control law, by using Theorem 1, we first consider stability of the LTTHS. The desired trajectory is periodic with a period of $5 \tau$, but due to its point symmetry with respect to the origin, it is possible to assess stability of the LTTHS corresponding to the considered system by only looking at the evolution from one post-impact position and velocity to the next. When applying a feedback input of the form (31) (with constant gain $K=K(t, j)$ ) to this linearized system, the state after the $j$-th impact and that just after the next are related to each other by $z\left(\tau_{j+1}, j+1\right)=$ $G(j) \exp ((A+B K) \tau) z\left(\tau_{j}, j\right)$, with $G(j)$ given by (24), combined with $\dot{\gamma}_{\alpha}^{-}=\mathbf{D}_{1} \gamma_{\alpha}^{-} \cdot f^{-}, \dot{g}^{-}=\mathbf{D}_{1} g^{-} \cdot f^{-}$,

$$
\begin{aligned}
f^{+} & =\left[\begin{array}{llll}
\alpha_{3}^{+} & \alpha_{4}^{+} & \mu_{1}^{+} & \mu_{2}^{+}
\end{array}\right]^{T}, \\
f^{-} & =\left[\begin{array}{llll}
\alpha_{3}^{-} & \alpha_{4}^{-} & \mu_{1}^{-} & \mu_{2}^{-}
\end{array}\right]^{T}, \\
\mathbf{D}_{1} \gamma_{\alpha}^{-} & =\left[\begin{array}{llll}
-2 \alpha_{1}^{-} & -2 \alpha_{2}^{-} & 0 & 0
\end{array}\right], \\
\mathbf{D}_{1} g^{-} & =\left[\begin{array}{cccc}
1 & 0 & 0 & 0 \\
0 & 1 & 0 & 0 \\
d g_{31} & d g_{32} & d g_{33} & d g_{34} \\
d g_{41} & d g_{42} & d g_{43} & d g_{44}
\end{array}\right],
\end{aligned}
$$

where

$$
\begin{aligned}
d g_{31} & =-2 e \alpha_{1}^{-} \alpha_{3}^{-}-(1+e) \alpha_{2}^{-} \alpha_{4}^{-} \\
d g_{32} & =2 \alpha_{2}^{-} \alpha_{3}^{-}-(1+e) \alpha_{1}^{-} \alpha_{4}^{-} \\
d g_{33} & =\left(\alpha_{2}^{-}\right)^{2}-e\left(\alpha_{1}^{-}\right)^{2} \\
d g_{34} & =-(1+e) \alpha_{1}^{-} \alpha_{2}^{-} \\
d g_{41} & =2 \alpha_{1}^{-} \alpha_{4}^{-}-(1+e) \alpha_{2}^{-} \alpha_{3}^{-} \\
d g_{42} & =-(1+e) \alpha_{1}^{-} \alpha_{3}^{-}-2 e \alpha_{2}^{-} \alpha_{4}^{-} \\
d g_{43} & =-(1+e) \alpha_{1}^{-} \alpha_{2}^{-} \\
d g_{44} & =\left(\alpha_{1}^{-}\right)^{2}-e\left(\alpha_{2}^{-}\right)^{2}
\end{aligned}
$$

and in which $\alpha_{s}^{+}=\alpha_{s}((j+1) \tau, j+1)$ and $\alpha_{s}^{-}=\alpha_{s}((j+$ 1) $\tau, j$ ) denote the right, respectively, left limit of the $s$-th state of the reference trajectory at time $t=(j+1) \tau$. Similarly, $\mu_{s}^{+}$and $\mu_{s}^{-}$denote the right and left limits (that are in this case the same), respectively, of the $s$-th reference input at that time. It follows that the LTTHS is asymptotically stable if all eigenvalues of the matrix $G(j) \exp ((A+B K) \tau)$ are within the unit circle in the complex plane. When taking a feedback gain of the form $K=\left[\begin{array}{llllllll}\beta & 0 & 2 \sqrt{\beta} & 0 ; & 0 & \beta & 0 & 2 \sqrt{\beta}\end{array}\right]$, it is found that the eigenvalues are within the unit circle when $\beta>0.393$. Applying Theorem 1, we therefore conclude that $\alpha$ is asymptotically stable for the cl-NSTHS (2), (32), (35), (36) for such choice of feedback gain. A solution to the clNSTHS (with constant gain extensions) for $\beta=3$ and initial condition $x_{0}=0$ is depicted in Fig. 4. The figure shows that the solution indeed converges towards the reference state-input trajectory $(\alpha(t, j), \mu(t, j))$, even for this large initial error. 


\section{Conclusions}

In this work, a notion of asymptotic stability and an associated stability analysis for discontinuous trajectories of hybrid systems with state-triggered jumps are detailed. The results have also a direct applicability to the related problem of trajectory tracking.

Asymptotic stability is defined by making use of a notion of error that allows for the comparison of two nearby discontinuous trajectories, even when there is a time mismatch between the jumps of both trajectories. It is shown that asymptotic stability of a discontinuous trajectory of the hybrid system with state-triggered jumps is guaranteed when an associate timetriggered linear system is uniformly asymptotically stable. As this linear system jumps at the same times as the reference trajectory, the design of a stabilizing feedback and stability analysis is greatly simplified. A study of (numerical) methods to estimate the associated region of attraction is left for future research. The results of this work are illustrated by means of a tracking example for a mechanical system with unilateral constraint and partially elastic impacts.

In a series of related publications, the tracking strategy based on reference extensions has been given the name of reference spreading control and has been applied to more complex systems (such as, e.g., a multi-body humanoid model) and even for the case where the constrained state space after each jump has a different dimension, representing an example of effective tracking for a multi-domain hybrid system. Investigation of the stability of these more challenging cases will be presented in future publications..

\section{APPENDIX A \\ PROOF OF LEMMA 1}

The proof of Lemma 1 is split in two parts. First, we show that the solution $x(t, j)$ to the NSTHS (6) from initial condition $x\left(t_{0}, 0\right)=x_{0}$ is defined for all $t \in\left[t_{0}, T\right]$ as long as $x_{0}$ is sufficiently close to $\alpha_{0}$. Then, we show that the bracketing condition (34) is satisfied as long as this neighborhood is chosen small enough, due to a continuity argument. This sufficiently small neighborhood is, in essence, what defines the function $\delta_{0}$ at $T$.

Due to Assumption $1, j_{\alpha}(T)$ is finite for any $T>t_{0}$. The existence of a solution to (6) up to time $T$ is straightforwardly guaranteed if, for every $j \in\left\{1,2, \ldots, j_{\alpha}(T)\right\}$ and in a neighborhood of $\alpha_{0}$, we can define an event-time function $x_{0} \mapsto t_{j}$ that is continuously differentiable, where $t_{j}\left(x_{0}\right)=\tau_{j}$ whenever $x_{0}=\alpha_{0}$. Indeed, if these functions $x_{0} \mapsto t_{j}, j \in\left\{1,2, \ldots, j_{\alpha}(T)\right\}$ exist, the flow of the NSTHS (6) is a composition of continuously differentiable jump maps (due to Assumption 4) with continuously differentiable continuous-time flows on the time intervals $\left[t_{j-1}\left(x_{0}\right), t_{j}\left(x_{0}\right)\right]$, $j \in\left\{1,2, \ldots, j_{\alpha}(T)-1\right\}$, terminated by a continuous-time flow over $\left[t_{j_{\alpha}(T)-1}\left(x_{0}\right), T\right]$, when $t_{j_{\alpha}(T)}\left(x_{0}\right) \geq T$, or over $\left[t_{j_{\alpha}(T)-1}\left(x_{0}\right), t_{j_{\alpha}(T)}\left(x_{0}\right)\right]$ followed by a jump and another flow phase over $\left[t_{j_{\alpha}(T)}\left(x_{0}\right), T\right]$, when $t_{j_{\alpha}(T)}\left(x_{0}\right)<T$.

Due to Assumption 2, there exists a guard function $\gamma_{\alpha}$ that implicitly defines the jump set in a neighborhood of $\alpha$.
Therefore, if the event time functions $x_{0} \mapsto t_{j}$ exist, they have to satisfy the implicit conditions

$$
\gamma_{\alpha}\left(x\left(t_{j+1}\left(x_{0}\right), j\right), t_{j+1}\left(x_{0}\right), j\right)=0
$$

for $j \in\left\{0,1, \ldots, j_{\alpha}(T)-1\right\}$, where

$$
\begin{aligned}
x\left(t_{j+1}\left(x_{0}\right), j\right): & =\varphi_{j}\left(t_{j+1}\left(x_{0}\right), t_{j}\left(x_{0}\right), \phi_{\mathcal{H}}\left(t_{j}\left(x_{0}\right), t_{0}, x_{0}\right)\right) \\
& =\varphi_{j}\left(t_{j+1}\left(x_{0}\right), t_{j}\left(x_{0}\right), x\left(t_{j}\left(x_{0}\right), j\right)\right) .
\end{aligned}
$$

Note that Assumption 6 guarantees that the flow $\varphi_{j}$ as used above is defined whenever $x_{0}$ is sufficiently close to $\alpha_{0}$. By definition of $\alpha$ and $\gamma_{\alpha}$, we know that (37) holds at least when we pose $x_{0}=\alpha_{0}$ and $t_{j}\left(\alpha_{0}\right)=\tau_{j}$. Using (38), the implicit condition (37) can equivalently be rewritten as

$M_{j+1}\left(x_{0}, t\right):=\gamma_{\alpha}\left(\varphi_{j}\left(t, t_{j}\left(x_{0}\right), x\left(t_{j}\left(x_{0}\right), j\right)\right), t, j\right)=0$.

We aim to prove that, for each $j, t$ in (39) is a function of $x_{0}$, i.e., that $t=t_{j+1}\left(x_{0}\right)$. The transversality condition provided in Assumption 3 guarantees that one can apply the implicit function theorem for each of the implicit conditions in (39) and conclude that all the functions $x_{0} \mapsto t_{j}$ are continuously differentiable for a sufficiently small neighborhood of $\alpha_{0}$.

More precisely, one can employ a proof by induction showing that $x_{0} \mapsto t_{1}$ is continuously differentiable (base induction) and that $x_{0} \mapsto t_{j}$ being continuously differentiable implies $x_{0} \mapsto t_{j+1}$ to be continuously differentiable (induction step). The base induction has been proven in [19], while it is straightforward to show, that $x_{0} \mapsto t_{j}$ being continuously differentiable implies that $M_{j+1}$ is continuously differentiable in a neighborhood of $\left(\alpha_{0}, \tau_{j+1}\right)$ being the composition of continuously differentiable functions. Furthermore, as the partial derivative of $M_{j+1}$ in (39) with respect to $t$ evaluated at $\left(x_{0}, t\right)=\left(\alpha_{0}, \tau_{j+1}\right)$ is equivalent to the left-hand side of (16) and therefore nonzero by Assumption 3, the implicit function theorem can be applied to conclude that $t$ in (39) is indeed a function of $x_{0}$. There is however a fundamental limitation in carrying out this induction reasoning for an infinite number of jumps. In the induction step mentioned above, the neighborhood of $\alpha_{0}$ for which $t_{j}$ is defined can, in principle, become smaller and smaller as $j$ is increased and, in the worst case, ceases to exist if no other conditions are imposed (this corresponds to a situation where the intersection of an infinite number of open sets containing $\alpha_{0}$ just ends up in the closed set containing just the point itself). This is why, with the given assumptions, the statement of this lemma holds just for any finite value of $T$, but not for $T=\infty$. We will explain in the proof of Theorem 1 how to overcome this limitation by adding an extra condition on the solutions of the cl-LTTHS. This concludes the first part of the proof.

We now prove the existence of $\delta_{0}(T)>0$ to satisfy the bracketing condition (34). As the functions $x_{0} \mapsto t_{j}$ are continuous and we are considering the finite set $j \in$ $\left\{1, \ldots, j_{\alpha}(T)-1\right\}$, we can find a closed ball around $\alpha_{0}$ with radius $\delta_{0}(T)$ that is contained in each domain of definition of the functions $x_{0} \mapsto t_{j}$ and such that each function $x_{0} \mapsto t_{j}$ is contained in the interval $\left[\tau_{j-1}, \tau_{j+1}\right]$. 


\section{APPENDIX B \\ PROOF OF THEOREM 1}

The proof of Theorem 1 is divided in two steps. First, a Nonlinear Time-Triggered Hybrid System (NTTHS) related to the NSTHS will be introduced. The NTTHS has $\alpha(t, j)$ as a solution and the LTTHS is its linearization as shown in Appendix D. Fig. 5 schematically depicts the structure of the proof and the role of the NTTHS therein.

\section{A. Nonlinear time-triggered hybrid system (NTTHS)}

The NTTHS, introduced below, always jumps at the times $\tau_{j}$ of the reference $\alpha$ and, as will be clarified below, its solutions coincide with solutions of the NSTHS for continuous-times sufficiently far away from the reference jump times.

When we start with a state-input trajectory $(\alpha(t, j), \mu(t, j))$ of (2) and slightly change its initial condition or input, we typically obtain a trajectory $x(t, j)$ that jumps at different times than the reference jump times $\tau_{j}$. This has been shown already schematically in Fig. 2. We illustrate this phenomenon in more detail in Fig. 6 focussing on a single jump. In constructing the NTTHS, the procedure is to replace the trajectory of (2) from initial condition $x\left(t_{0}, 0\right)=x_{0}$ between the time instances $t_{j}$ and $\tau_{j}$ with a new trajectory that always jumps at time $\tau_{j}$, as illustrated in Fig. 6, where the trajectory of the NTTHS is denoted as $x_{T T}$. This construction is related to the concept of zero-time discontinuity mapping (ZDM) and Poincaré discontinuity mapping (PDM) in [29, Section 6.2]. The trajectory $x_{T T}$ near $\tau_{j+1}$ is attained by flowing according to the vector field $f(x, u, t, j)$ similarly as the reference trajectory up to $\tau_{j+1}$ and after the time $\tau_{j+1}$ by flowing according to the vector field $f(x, u, t, j+1)$. A suitable jump

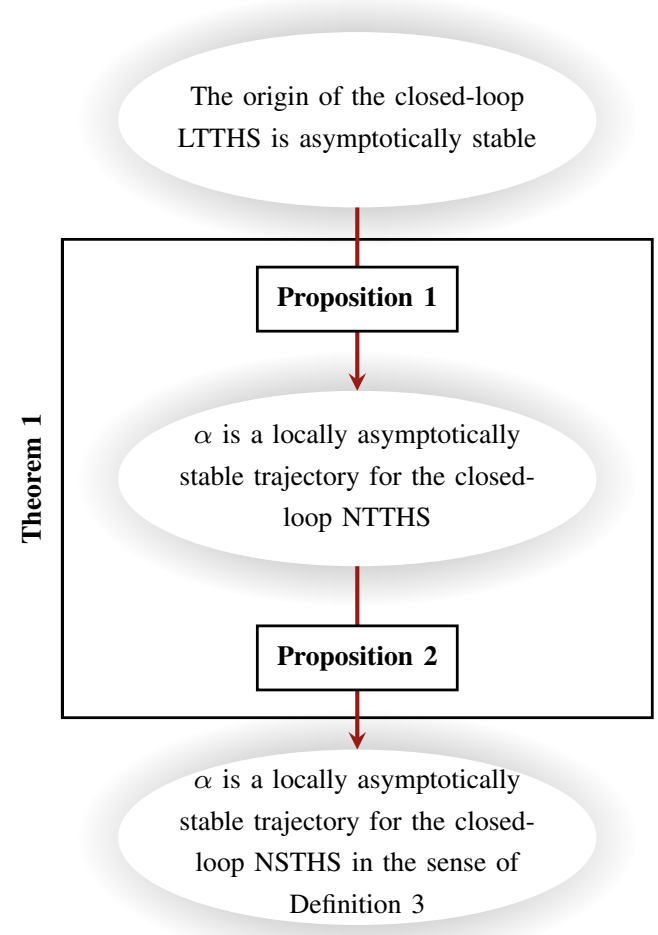

Fig. 5. Subdivision of Theorem 1 in two implication steps, by introducing the NTTHS and Propositions 1 and 2.

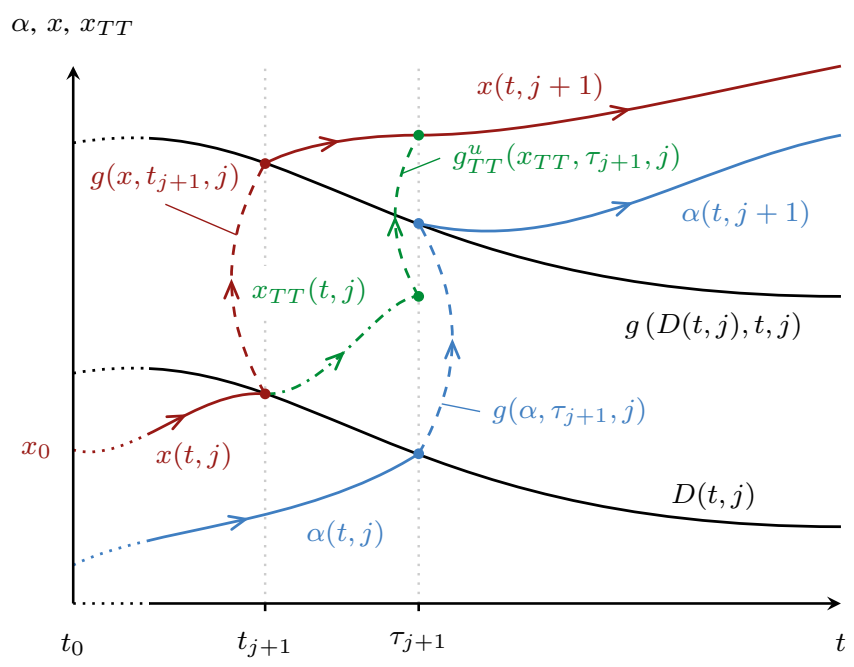

Fig. 6. Relation between trajectory $x(t, j)$ of the NSTHS, corresponding reference $\alpha(t, j)$, and trajectory $x_{T T}(t, j)$ of the NTTHS for jump $(j+1)$.

map is applied at the nominal event time $\tau_{j+1}$ such that it maps the trajectory $x_{T T}(t, j)$ back to the trajectory $x(t, j)$ at the end of this time mismatch period $\left(\left[t_{j+1}, \tau_{j+1}\right]\right.$ or $\left.\left[\tau_{j+1}, t_{j+1}\right]\right)$.

In order to define this jump map, we denote by $\varphi_{j}^{u}(t, \tau, x)$ the state evolution according to vector field $f$ for jump counter $j$ at time $t$ with initial condition $x$ at time $\tau$ and a given input curve $u(t, j)$. Note that $t \leq \tau$ implies integration backwards in time and that this operator is different for different input curves. The NTTHS, with state $x_{T T}$, is defined as follows.

Definition 7 (NTTHS). The nonlinear time-triggered hybrid system is given by

$$
\begin{array}{lll}
\dot{x}_{T T}=f\left(x_{T T}, u(t, j), t, j\right) & & (t, j) \in \operatorname{dom} \alpha \\
x_{T T}^{+}=g_{T T}^{u}\left(x_{T T}^{-}, t, j\right) & & (t, j) \in E_{\alpha},
\end{array}
$$

with initial condition $x_{T T}\left(t_{0}, 0\right)=x_{0}$, where $x_{T T}^{+}=$ $x_{T T}(t, j+1), x_{T T}^{-}=x_{T T}(t, j)$, and the jump map $g_{T T}^{u}\left(x_{T T}, t, j\right)$, with $(t, j) \in E_{\alpha}$, is given by

$$
\varphi_{j+1}^{u}\left(t, t_{j+1}, g\left(\varphi_{j}^{u}\left(t_{j+1}, t, x_{T T}\right), t_{j+1}, j\right)\right),
$$

where $t_{j+1}$ is the $(j+1)$-th jump time of the solution $x(t, j)$ of the NSTHS (2) starting from the initial condition $x_{0}$.

The jump map $g_{T T}^{u}\left(x_{T T}, \tau_{j+1}, j\right)$ can be defined whenever $t_{j+1}$ is defined, i.e. when the trajectory $x$ of the NSTHS with initial condition $x\left(t_{0}\right)=x_{0}$ and chosen input $u(t, j)$ will experience the $(j+1)$-th jump (see Fig. 6). This property is satisfied if $x_{0}$ is close enough to $\alpha_{0}$ and follows from Lemma 1. More details are provided in the proof of Proposition 1.

Furthermore, since the NTTHS jumps at the same times as the reference, the time domain of its solution $x_{T T}(t, j)$ is the same as that of the reference trajectory, i.e. dom $x_{T T}=$ dom $\alpha$, as illustrated in Fig. 7. This figure also illustrates the time-triggered error $e_{T T}(t, j):=x_{T T}(t, j)-\alpha(t, j)$ (compare with Fig. 2) and their hybrid time domains, $e_{T T}$ being the error for trajectories with jumps at fixed time instants.

When the input $u$ is given by

$$
u=\kappa\left(x_{T T}, t, j\right)
$$


as in (5), we obtain the closed-loop NTTHS (or cl-NTTHS)

$$
\begin{array}{ll}
\dot{x}_{T T}=f_{c l}\left(x_{T T}, t, j\right) & (t, j) \in \operatorname{dom} \alpha, \\
x_{T T}^{+}=g_{T T}\left(x_{T T}^{-}, t, j\right) & (t, j) \in E_{\alpha},
\end{array}
$$

with $x_{T T}\left(t_{0}, 0\right)=x_{0}$ and where the jump map $g_{T T}\left(x_{T T}, t, j\right)$ is given by

$$
\varphi_{j+1}\left(t, t_{j+1}, g\left(\varphi_{j}\left(t_{j+1}, t, x_{T T}(t, j)\right), t_{j+1}, j\right)\right)
$$

with $\varphi_{j}$ the closed-loop flow as in (41) with $u$ as in (42). More specifically, the cl-NTTHS that we will use in the proof of Theorem 1 is the one employing the affine feedback law

$$
u(t, j)=\mu(t, j)+K(t, j)\left(x_{T T}(t, j)-\alpha(t, j)\right) .
$$

Note that (45) is the same as (32), but that in the latter no "bars" are needed on top of $K$ and $\alpha$ as the controller will only be used for the hybrid times $(t, j) \in \operatorname{dom} x_{T T}=\operatorname{dom} \alpha$.

\section{B. Proposition 1 and its proof}

As mentioned at the beginning of this appendix, a key property in the proof of Theorem 1 is that the linearization of the NTTHS in Definition 7 is the LTTHS provided by Definition 4. Given $\alpha$, solutions to the NTTHS and LTTHS clearly have the same hybrid time domain. Here we prove that uniform asymptotic stability of the closed-loop linearization implies that the reference trajectory $\alpha(t, j)$ is a locally asymptotically stable solution to the cl-NTTHS.

Proposition 1. A trajectory $(\alpha(t, j), \mu(t, j))$ of the NSTHS (6), (32) satisfying Assumptions 1, 2, 3, 4, 5, and 6, is an asymptotically stable trajectory of the cl-NTTHS (43), (45), if the associated cl-LTTHS (21)-(31) is uniformly asymptotically stable.

Proof. As both cl-LTTHS and cl-NTTHS are time-triggered and each jump event corresponds to a jump event of $\alpha$, we can employ the jump counter $j_{\alpha}: \mathbb{R} \rightarrow \mathbb{N}$ of Definition 6 to simplify the notation within this proof. To this end, with a slight abuse of notation, we will write $\alpha(t), z(t), x_{T T}(t)$, etc. to mean $\alpha\left(t, j_{\alpha}(t)\right), z\left(t, j_{\alpha}(t)\right), x_{T T}\left(t, j_{\alpha}(t)\right)$, etc. At the

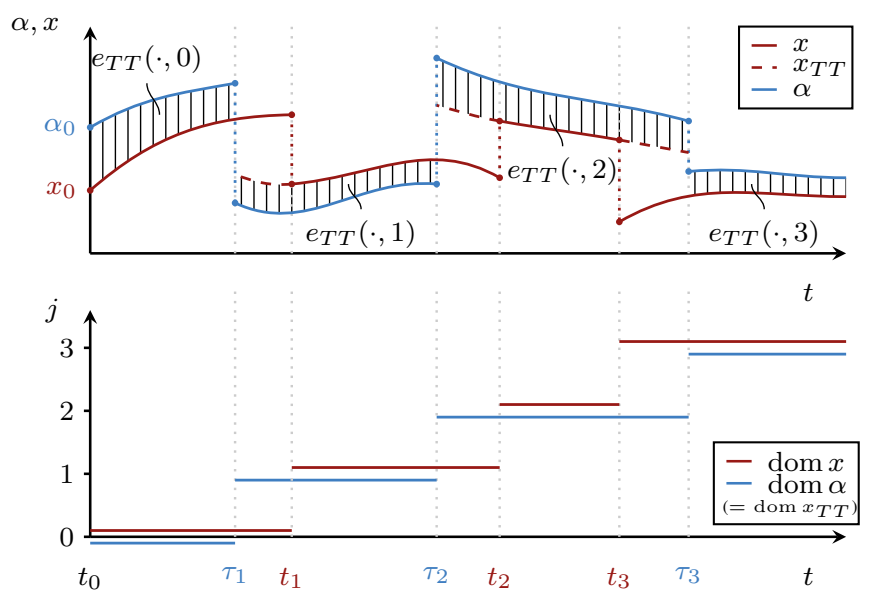

Fig. 7. The classical error for the time-triggered hybrid system $\left(e_{T T}(t, j)=\right.$ $\left.x_{T T}(t, j)-\alpha(t, j)\right)$ and the hybrid time domains of the corresponding required trajectories. event times $\tau_{j}$, we will write $\alpha^{+}\left(\tau_{j}\right)$ and $\alpha^{-}\left(\tau_{j}\right)$ to indicate $\alpha\left(\tau_{j}, j_{\alpha}\left(\tau_{j}\right)\right)$ and $\alpha\left(\tau_{j}, j_{\alpha}\left(\tau_{j}\right)-1\right)$, respectively. Similarly, we will employ the + and - notation for other signals.

Our goal is to conclude local asymptotic stability of $\alpha$ for the cl-NTTHS. To this end, let us consider the time-triggered error $e_{T T}(t)$ between the state of the cl-NTTHS and the reference $\alpha$. The error $e_{T T}$ satisfies the hybrid dynamics

$$
\begin{aligned}
& \dot{e}_{T T}=A_{c l}(t) e_{T T}+r_{1}\left(e_{T T}, t\right), \\
& e_{T T}^{+}=G(j) e_{T T}^{-}+r_{2}\left(e_{T T}^{-}, j\right),
\end{aligned}
$$

with $j=j_{\alpha}(t)-1, e_{T T}\left(t_{0}\right)=z_{0}$, and where the matrices $A_{c l}$ and $G$ and the residuals $r_{1}$ and $r_{2}$ are defined below. In (46), $e_{T T}$ is obtained by alternating state resets according to the jump map with integrations of the ODE until $t$ equals $\tau_{j+1}$. In (46), $A_{c l}(t):=A\left(t, j_{\alpha}(t)\right)+B\left(t, j_{\alpha}(t)\right) K\left(t, j_{\alpha}(t)\right)$ and $G$ is given by (24), therefore corresponding to the clLTTHS. The residuals $r_{1}$ and $r_{2}$ are the higher-order terms of the vector field and jump map of the time-triggered error dynamics associated to the cl-NTTHS, namely

$$
\begin{aligned}
r_{1}\left(e_{T T}, t\right) & :=f_{c l}\left(\alpha(t)+e_{T T}, t, j_{\alpha}(t)\right) \\
& -f_{c l}\left(\alpha(t), t, j_{\alpha}(t)\right)-A_{c l}(t) e_{T T} \\
r_{2}\left(e_{T T}, j\right) & :=g_{T T}\left(\alpha^{-}\left(\tau_{j+1}\right)+e_{T T}^{-}, \tau_{j+1}, j\right) \\
& -g_{T T}\left(\alpha^{-}\left(\tau_{j+1}\right), \tau_{j+1}, j\right)-G(j) e_{T T}^{-} .
\end{aligned}
$$

The origin $e_{T T}=0$ is an equilibrium point for (46) and for the cl-LTTHS. Both these hybrid systems jump at the same fixed time instants $\tau_{j}$, known in advance. For such class of systems, denoting generically the system state with $y$, uniform asymptotic stability implies that for an arbitrary $\varepsilon_{T T}>0$ and $t_{S} \geq t_{0}$ there exists a $\delta_{T T}$ such that $\left\|y\left(t_{S}\right)\right\|<\delta_{T T}$ implies $\|y(t)\|<\varepsilon_{T T}$ for all $t \geq t_{S}$ and that, furthermore, $\lim _{t \rightarrow \infty}|y(t)|=0$ (see, e.g., [27, Section 3.1]). We aim to show that uniform asymptotic stability of the cl-LTTHS implies that the origin $e_{T T}=0$ is uniformly locally asymptotically stable for (46). Let us now consider two cases:

CASE 1: Consider first the case where the number of events $J_{\alpha}-1$ is infinite. Denote with $L_{\kappa}>0$ the Lipschitz constant for which $\kappa$ in (32) satisfies $\|\kappa(x, t, j)-\kappa(y, t, j)\|<L_{\kappa} \| x-$ $y \|, \forall(t, j) \in \bar{I}_{\alpha}$, that is $L_{\kappa}=\sup _{(t, j) \in \bar{I}_{\alpha}}\|\bar{K}(t, j)\|$. From Assumption 5, for each time interval $I_{\alpha}^{j}=\left[\tau_{j}, \tau_{j+1}\right], j=$ $0,1,2, \ldots$, the growth of the solution can be bounded (see, e.g., [30, Corollary 6.4]) according to

$$
\|y(t)\| \leq \exp \left(L_{c l}\left(t-\tau_{j}\right)\right)\left\|y^{+}\left(\tau_{j}\right)\right\|, \quad \tau_{j} \leq t \leq \tau_{j+1}
$$

with $L_{c l}=L\left(1+L_{\kappa}\right)$. Clearly, we require $\left\|y^{+}\left(\tau_{j}\right)\right\|$ to be also sufficiently small to keep $\|y(t)\|$ within the region where the vector field $f$ is uniformly Lipschitz. When the continuous dynamics satisfies an exponential bound as in (49), asymptotic stability of a time-triggered hybrid system is equivalent to the asymptotic stability of the discrete time system that originates from only considering the state at the event times $\tau_{j}$ [31]. Recall that $\tau_{j+1}-\tau_{j}$ is uniformly bounded from below and from above (bounded inter-jump time) due to Assumption 1.

In our case, the local asymptotic stability of the cl-NTTHS follows directly from uniform asymptotic stability (for linear systems, equivalent to exponential stability) of the cl-LTTHS, 


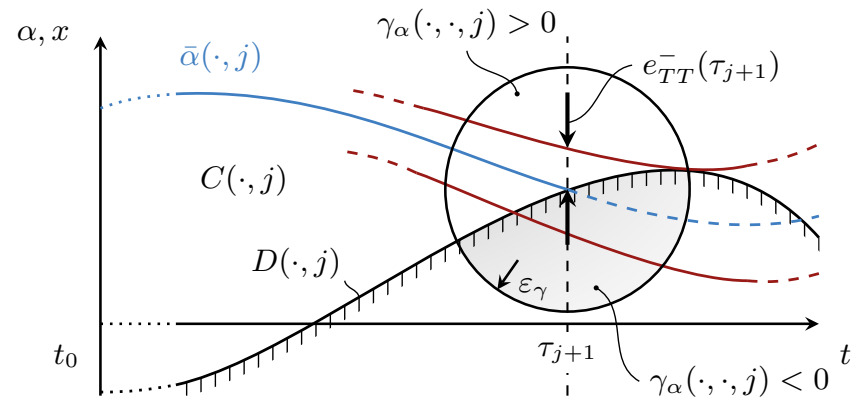

Fig. 8. Illustration of the fact that the transversality assumption (Assumption 3) implies a relation between state error and event time mismatch.

by considering their respective associated discrete time systems at the nominal event times. This statement is based on the discrete time version of [32, Theorem 4.13]. This concludes the proof for the case where $J_{\alpha}=\infty$.

CASE 2: When the number of events $J_{\alpha}-1$ is finite, it suffices to consider the stability of the continuous time dynamics after the last event. This is valid because, from (49) and continuity of $g_{T T}$ with respect to $x_{T T}$, an exponential bound on the error $\left\|e_{T T}\right\|$ of the cl-NTTHS in the finite time interval $\left[t_{0}, \tau_{J_{\alpha}-1}\right]$ can be found (in particular, for every $\varepsilon>0$ and $t_{0} \leq t_{S} \leq \tau_{J_{\alpha}-1}$ there is a $\delta>0$ such that $\left\|e_{T T}\left(t_{S}\right)\right\|<\delta$ implies $\left.\left\|e_{T T}\left(\tau_{J_{\alpha}-1}\right)\right\|<\varepsilon\right)$. It thus suffices to consider the last flow phase when $t \geq \tau_{J_{\alpha}-1}$.

As the cl-LTTHS is, by assumption, exponentially stable and it is the linearization of the error system (46), specifically in the time interval $\left[\tau_{J_{\alpha}-1}, \infty\right)$, the origin of the error system is (locally) asymptotically stable [32, Theorem 4.13] and, equivalently, $\alpha$ is an asymptotically stable trajectory of the cl-NTTHS. This concludes the proof for finite $J_{\alpha}$.

So far, it has been tacitly assumed that the solution of the NTTHS exists for all $t>t_{0}$. However, one has actually to prove this result based on the assumptions in the proposition statement. For the NTTHS to be defined, the jump map $g_{T T}$ needs to be defined for all jumps, which in turn requires that the NSTHS corresponding to the NTTHS experiences those jumps as well as we discussed after (41).

From the proof of Lemma 1 and, in particular, from the continuity of the map $t_{j}\left(x_{0}\right)$, the $j$-th jump of the NSTHS occurs close to the $j$-th jump time of $\alpha$ as long as $\left\|e_{T T}\left(t_{0}\right)\right\|$ is chosen in a sufficiently small neighborhood of zero (equivalently, $x_{T T}\left(t_{0}, 0\right)$ is chosen sufficiently close to $\left.\alpha_{0}\right)$. This property is illustrated in Fig. 8. However, a uniform (i.e., independent of $j$ ) neighborhood is not guaranteed to exist when $\alpha$ has an infinite number of jumps (cf. proof of Lemma 1). Assuming the exponential stability of the cl-LTTHS, though, guarantees the existence of such a uniform neighborhood.

We start by noting that the $j$-th time-triggered jump map of (46) is defined as long as $e_{T T}^{-}\left(\tau_{j}\right)$ is sufficiently small, following from the fact that the $j$-th jump occurs for the original NSTHS if $x(t, j)$ is sufficiently close to $\alpha(t, j)$ as discussed in the proof of Lemma $1 .\left\|e_{T T}^{-}\left(\tau_{j}\right)\right\|$ is required to be smaller than $\varepsilon_{\gamma}$ for example (cf. Assumption 2). Equivalently, the $j$-th time-triggered jump map $g_{T T}(\cdot, j)$ of the clNTTHS is defined as long as $x_{T T}^{-}\left(\tau_{j}\right)$ is sufficiently close to $\alpha^{-}\left(\tau_{j}\right):=\alpha\left(\tau_{j}, j-1\right)$. The proof for the existence of a neighborhood about the origin for $e_{T T}^{-}\left(\tau_{j}\right)$ where the time-triggered jump map is defined follows again from a straightforward application of the implicit function theorem employing the guard function $\gamma_{\alpha}(\cdot, \cdot, j)$. In particular, one can show that when $\left\|e_{T T}^{-}\left(\tau_{j}\right)\right\|=\left\|x_{T T}^{-}\left(\tau_{j}\right)-\alpha^{-}\left(\tau_{j}\right)\right\|$ is sufficiently small then $\left|t_{j, T T}-\tau_{j}\right|<S_{j}\left\|e_{T T}^{-}\left(\tau_{j}\right)\right\|$ where the function $t_{j, T T}:=t_{j, T T}(e)$ represents the event time of the cl-NSTHS corresponding to the time-triggered error trajectory with value $e=e_{T T}^{-}\left(\tau_{j}\right)$ at $\tau_{j}$ and $S_{j}$ a constant that depends on the continuous-time closed-loop vector field $f_{c l}$, guard function $\gamma_{\alpha}$, and the reference trajectory $\alpha$.

In details, the function $t_{j, T T}(e)$ is implicitly defined as $H_{j}(t, e):=\gamma_{\alpha}\left(\varphi_{j-1}\left(t, \tau_{j}, \alpha\left(\tau_{j}, j-1\right)+e\right), t, j-1\right)=0$ and Assumption 3 provides the sufficient condition for the validity of the application of the implicit function theorem. Again, note that due to Assumption 6 the flow $\varphi_{j-1}$ in $H_{j}$ is defined as long as it is sufficiently close to the reference trajectory. Finally, because $t_{j, T T}(e)=\tau_{j}+\mathbf{D} t_{j, T T}(0) \cdot e+o(|e|)$, by choosing $S_{j}>\left\|\mathbf{D} t_{j, T T}(0)\right\|>0$, one obtains $\left|t_{j, T T}-\tau_{j}\right|<$ $S_{j}\|e\|$ for sufficiently small $e$. A uniform bound on the maximum time shift of the form $\left|t_{j, T T}-\tau_{j}\right|<S\|e\|$, valid for all (possibly infinite) $j$, follows from the uniform bound on the derivative of $\gamma_{\alpha}$ in Assumption 2. It is straightforward to show that $\left\|\mathbf{D}_{1} t_{j, T T}(0)\right\|=\left\|\mathbf{D}_{1} H_{j}\left(\tau_{j}, 0\right)\right\|^{-1}\left\|\mathbf{D}_{2} H_{j}\left(\tau_{j}, 0\right)\right\| \leq$ $c_{1} / c_{2}$ (see Assumptions 2 and 3 ).

From the above, one concludes that as long as $e_{T T}(t)$ remains within a predetermined sufficiently small neighborhood of the origin, then the (possibly infinite number of) jump maps $g_{T T}$ are all defined (implying $J_{x} \geq J_{\alpha}$ ), with the difference between the jump times $t_{j}$ of the cl-NSTHS and the nominal jump times $\tau_{j}$ becoming smaller as this neighborhood is chosen smaller. Uniform asymptotic stability of the LTTHS allows then to conclude that $e_{T T}(t)$, will remain within such a predetermined, sufficiently small, neighborhood where all jump maps $g_{T T}$ are defined as long as the initial condition $e_{T T}\left(t_{0}\right)$ is chosen sufficiently close to zero, ensuring the existence of $e_{T T}(t)$ for $t \rightarrow \infty$. This concludes the proof of the proposition.

\section{Proposition 2 and its proof}

Proposition 2. Let $(\alpha(t, j), \mu(t, j))$ be a trajectory of the NSTHS (6) satisfying Assumptions 1, 2, 3, 4, 5, and 6. If $\alpha(t, j)$ is an asymptotically stable solution of the cl-NTTHS (43), (45), then it is also an asymptotically stable solution of the cl-NSTHS (6), (32), in the sense of Definition 3.

Proof. By construction, except in suitable neighborhoods of the event times $\tau_{j}$, the solution of the cl-NTTHS is identical, when it exists, to the solution of the cl-NSTHS. As $\alpha$ is assumed to be asymptotically stable for the cl-NTTHS, the solution of the cl-NTTHS exists for all $t \geq t_{0}$ as long as the corresponding initial condition is taken sufficiently close to $\alpha\left(t_{0}, 0\right)$. This follows, in particular, from the uniform bound

$$
\begin{aligned}
\left|t_{j}-\tau_{j}\right| & <S\left\|e_{T T}\left(\tau_{j}, j-1\right)\right\| \\
& =S\left\|x_{T T}\left(\tau_{j}, j-1\right)-\alpha\left(\tau_{j}, j-1\right)\right\|
\end{aligned}
$$

obtained in the proof of Proposition 1, with $S$ a suitable strictly positive constant. From the proof of Proposition 1 and 
Assumption 6 furthermore follows that, if the initial condition of the cl-NSTHS is taken sufficiently close to $\alpha\left(t_{0}, 0\right)$, it is guaranteed that the number of events $J_{x}-1$ of the cl-NSTHS (equivalently, of the cl-NTTHS) equals the number of events $J_{\alpha}-1$ of the reference. The equality $J_{x}=J_{\alpha}$ is the first condition that $\alpha$ has to satisfy for being locally asymptotically stable for the cl-NSTHS, in the sense of Definition 3.

What is left to be shown in this proof is therefore that the other two conditions of Definition 3 are fulfilled. Namely, guaranteeing that for an arbitrary $\varepsilon>0$, there exists a $\delta=\delta(\varepsilon)$ such that $\left\|x\left(t_{0}, 0\right)-\alpha\left(t_{0}, 0\right)\right\|<\delta$ implies $\left|t_{j}-\tau_{j}\right|<\varepsilon$ and $\|x(t, j)-\bar{\alpha}(t, j)\|<\varepsilon$ for all $(t, j) \in \operatorname{dom} x$ and that both quantities converge to zero as $t \rightarrow \infty$. We will show that taking $\varepsilon_{T T}=\varepsilon_{T T}(\varepsilon)$ such that

$$
\max \left(\varepsilon_{T T}, S \varepsilon_{T T}, \exp \left(L_{c l} S \varepsilon_{T T}\right) \varepsilon_{T T}\right) \leq \varepsilon
$$

with $S>0$ as in (50) and $L_{c l}=L\left(1+L_{\kappa}\right)>0$ as in (49) (cf. Assumption 5) and $\delta:=\delta_{T T}\left(\varepsilon_{T T}(\varepsilon)\right)$ will indeed satisfy the above stability conditions.

First, for a given $\varepsilon$, if $\varepsilon_{T T}$ is chosen according to (51) then local asymptotic stability of $\alpha$ for the cl-NTTHS ensures the existence of a neighborhood of $\alpha\left(t_{0}, 0\right)$ containing a ball of radius $\delta=\delta(\varepsilon)$ such that the corresponding trajectories of the cl-NSTHS satisfy the bound $\left|t_{j}-\tau_{j}\right|<S \varepsilon_{T T} \leq \varepsilon$, as requested by Definition 3.

Second, from (50), (51), and local asymptotic stability of the cl-NTTHS, one concludes immediately that $\| x_{T T}(t, j)-$ $\alpha(t, j) \|<\varepsilon_{T T} \leq \varepsilon$ for every time interval $I_{j}^{\alpha}$. However, in fact, we need to prove something stronger, namely that $\|x(t, j)-\bar{\alpha}(t, j)\|<\varepsilon$, for $t \in I_{x}^{j}=\left[t_{j-1}, t_{j}\right]$. In the simplest case, namely when $\tau_{j} \leq t_{j}$ and $t_{j+1} \leq \tau_{j+1}$, the condition is trivially satisfied on the $j$-th time interval $I_{j}^{x}$, because there $x(t, j)=x_{T T}(t, j)$ and $\bar{\alpha}(t, j)=\alpha(t, j)$. In general, one will have $t_{j} \leq \tau_{j}$ and/or $\tau_{j+1} \leq t_{j+1}$ : indeed, if the simplest case occurs in $j$-th interval, by construction, it will not occur in the $(j-1)$-th and $(j+1)$-th intervals. Fortunately, as in the proof of Proposition 1, we can make use of a uniform exponential bound on the growth of the solutions of locally Lipschitz vector fields, cf. (49). For a given initial condition $x\left(t_{0}, 0\right)$ within the ball of radius $\delta=\delta_{T T}\left(\varepsilon_{T T}(\varepsilon)\right)$ centered at $\alpha\left(t_{0}, 0\right)$, if $\tau_{j+1} \leq t_{j+1}$, then we can bound the solutions of the cl-NSTHS for $t \in\left[\tau_{j+1}, t_{j+1}\right]$ as

$$
\begin{aligned}
\|x(t, j)-\bar{\alpha}(t, j)\| & \leq \exp \left(L_{c l}\left(t-\tau_{j+1}\right)\right)\left\|e_{T T}\left(\tau_{j+1}, j\right)\right\| \\
& \leq \exp \left(L_{c l}\left(t-\tau_{j+1}\right)\right) \varepsilon_{T T}, \quad \text { (52) }
\end{aligned}
$$

where $L_{c l}=L\left(1+L_{\kappa}\right)$ follows from the upper bound $L$ on the Lipschitz constants as in Assumption 5 and the upper bound $L_{\kappa}$ on Lipschitz continuity of (32). Similarly, if $t_{j} \leq \tau_{j}$, then we can bound the solutions of cl-NSTHS for $t \in\left[t_{j}, \tau_{j}\right]$ as

$$
\begin{aligned}
\|x(t, j)-\bar{\alpha}(t, j)\| & \leq \exp \left(L_{c l}\left(\tau_{j}-t\right)\right)\left\|e_{T T}\left(\tau_{j}, j\right)\right\| \\
& \leq \exp \left(L_{c l}\left(\tau_{j}-t\right)\right) \varepsilon_{T T}
\end{aligned}
$$

Recalling (50) and for each $j$, the two equations above, due to (51), finally lead to $\|x(t, j)-\bar{\alpha}(t, j)\|<\exp \left(L S \varepsilon_{T T}\right) \varepsilon_{T T} \leq$ $\varepsilon$ for $t \in I_{x}^{j}=\left[t_{j-1}, t_{j}\right]$, as required in Definition 3 .

Convergence to zero of $\left\|t_{j}-\tau_{j}\right\|$ and $\|x(t, j)-\bar{\alpha}(t, j)\|$ for $t \rightarrow \infty$ follows from the convergence to zero of $\| x_{T T}\left(\tau_{j}, j\right)-$ $\alpha\left(\tau_{j}, j\right) \|$ as $j=j_{x}(t) \rightarrow \infty$. This concludes the proof.
It can be concluded that the trajectory $\alpha(t, j)$ is an asymptotically stable solution of the cl-NSTHS (6), (32) if it is an asymptotically stable trajectory for the cl-NTTHS (43) (Prop. 2), which is the case if the origin of the closed-loop linearization (21)-(31) about that trajectory, also referred to as the cl-LTTHS, is uniformly asymptotically stable (Prop. 1). These two steps together form the proof of Theorem 1.

\section{APPENDIX C}

\section{RELATION TO EXISTING DEFINITION OF STABILITY}

In this appendix, we will present a link with existing literature on hybrid system stability analysis. More precisely, we will show that uniform asymptotic stability of the origin of the cl-LTTHS (21)-(31) implies asymptotic stability (as defined in [13, Definition 2] and given below) of the reference trajectory $\alpha$ employing the distance function given in [13, Definition 1]. As this distance function is not designed to accommodate non-autonomous jump maps, flow sets, and jump sets, in this appendix, we consider state-triggered hybrid systems of the following form:

$$
\begin{aligned}
\dot{x} & =f(x, u, t, j) & & x \in C \\
x^{+} & =g\left(x^{-}\right) & & x \in D,
\end{aligned}
$$

with vector field $f(x, u, t, j): \mathbb{R}^{n} \times \mathbb{R}^{m} \times \mathbb{R} \times \mathbb{N} \rightarrow \mathbb{R}^{n}$, jump map $g: D \rightarrow \mathbb{R}^{n}$, and constant flow and jump sets $C \subseteq \mathbb{R}^{n}$ and $D \subseteq \partial C$, respectively. Moreover, for the sake of brevity, when we refer to $x(t)$ and $\alpha(t)$ in this appendix, one should read $x\left(t, j_{x}(t)\right)$ and $\alpha\left(t, j_{\alpha}(t)\right)$, respectively, where $j_{x}$ and $j_{\alpha}$ are given by Definition 6 in Section III-D for the trajectories $x$ and $\alpha$. In the following, by (asymptotic) stability in terms of the distance function $d$ we mean (cf. [13, Definition 2]

Definition 8. The trajectory $\alpha$ is stable with respect to the distance $d$ if for all $\varepsilon_{d}>0$, there exists a $\delta_{d}\left(\varepsilon_{d}\right)>0$ such that for every trajectory $x$ of (32), (54) satisfying $d\left(x_{0}, \alpha_{0}\right)<$ $\delta_{d}\left(\varepsilon_{d}\right)$, it holds that $d(x(t), \alpha(t))<\varepsilon_{d}$ for all $t \geq t_{0}$. If it moreover holds that $d(x(t), \alpha(t)) \rightarrow 0$ for $t \rightarrow \infty$, then the trajectory $\alpha$ is asymptotically stable with respect to $d$.

We can now formulate the following result.

Corollary 1. Adopt Assumptions 1-6. Let a state-input trajectory $(\alpha, \mu)$ be a solution to the cl-NSTHS (32), (54), starting from the initial condition $\alpha\left(t_{0}\right)=\alpha_{0} \in \operatorname{int} C \backslash g(D)$. Let the closed-loop vector field furthermore satisfy $\left\|f_{c l}(x, t)\right\|<F$ for some $F>0$ and for all $(x, t)$ in a neighborhood of the graph of $\alpha$. If the origin of the associated cl-LTTHS (21)-(31) is uniformly asymptotically stable, then, given any uniformly continuous distance function $d: \mathbb{R}^{n} \times \mathbb{R}^{n} \rightarrow \mathbb{R}_{\geq 0}$ that is compatible with (32), (54) in the sense of [13, Definition 1], the trajectory $\alpha$ of (32), (54) is asymptotically stable with respect to the distance d as given in Definition 8.

Proof. Stability of the trajectory $\alpha$ is guaranteed if A) for every $\varepsilon_{d}>0$, there exist $\varepsilon_{T T}\left(\varepsilon_{d}\right)>0$ and $\delta_{T T}\left(\varepsilon_{d}\right)$ such that, for all trajectories $x, x_{T T}$ (to (32), (54), respectively, the autonomous equivalent of (43)) with initial condition $x\left(t_{0}\right)=$ $x_{T T}\left(t_{0}\right)=x_{0}$ satisfying $\left.\| x_{0}-\alpha_{0}\right) \|<\delta_{T T}\left(\varepsilon_{d}\right)$ it holds that

$$
\left\|x_{T T}(t)-\alpha(t)\right\|<\varepsilon_{T T}\left(\varepsilon_{d}\right) \Longrightarrow d(x(t), \alpha(t))<\varepsilon_{d}
$$


and B), that for this $\delta_{T T}\left(\varepsilon_{d}\right)$, there exists a $\delta_{d}\left(\varepsilon_{d}\right)>0$ such that

$$
d\left(x_{0}, \alpha_{0}\right)<\delta_{d}\left(\varepsilon_{d}\right) \Longrightarrow\left\|x_{0}-\alpha_{0}\right\|<\delta_{T T}\left(\varepsilon_{d}\right) .
$$

In proving that condition A) is satisfied, we start by pointing out that, from Proposition $1, \alpha$ is an asymptotically stable solution to the cl-NTTHS (43), (45) since, by hypothesis in the theorem, the cl-LTTHS (40)-(31) is uniformly asymptotically stable. From this, we can conclude that for every $\varepsilon_{T T}>0$ there exists a $\delta_{T T}>0$ such that $\left\|x_{T T}\left(t_{0}\right)-\alpha\left(t_{0}\right)\right\|<\delta_{T T}$ implies that $\left\|x_{T T}(t)-\alpha(t)\right\|<\varepsilon_{T T}$ for all $t \geq t_{0}$. What remains to be proven is that if we arbitrarily fix $\varepsilon_{d}$, we can indeed find a $\varepsilon_{T T}$ such that (55) is satisfied. In doing so, we make a distinction between the times $t$ for which $\left(t, j_{\alpha}(t)\right) \in$ dom $x \cap$ dom $\alpha$, i.e. the times for which the trajectory $x$ and the reference $\alpha$ have encountered the same number of jumps, and the times $t$ for which this is not the case. In the former situation, we exploit uniform continuity of $d$, as well as the fact that $d(x, y)=0$ if $x=y$ [13, Definition 1], to infer that there exists a $\varepsilon_{T T}^{\prime}>0$ such that $\|x-y\|<\varepsilon_{T T}^{\prime}$ implies $d(x, y)<\varepsilon_{d}$. Exploiting that in this situation, for those specific times, as by construction, $x_{T T}(t)=x(t)$, we conclude that (55) clearly is satisfied whenever $\varepsilon_{T T}\left(\varepsilon_{d}\right) \leq \varepsilon_{T T}^{\prime}$.

Let us now look at the times for which $x$ has experienced more or less jumps than $\alpha$ has (i.e. the times $t$ for which $\left.j_{x}(t) \neq j_{\alpha}(t)\right)$ and consider an arbitrary event time $\tau_{j}$ of the reference. The $j$-th jump time "mismatch" interval we denote by $I_{\Delta}^{j}$ and define it as $I_{\Delta}^{j}:=\left(t_{j}, \tau_{j}\right)$ when $t_{j}<\tau_{j}$ and as $I_{\Delta}^{j}:=\left(\tau_{j}, t_{j}\right)$ in the case that $\tau_{j}<t_{j}$ (where we exploit the bracketing condition in Lemma 1). We remark that $d$ is invariant to jumps in $x$ as well as to jumps in $\alpha$ and it is a continuous function. It follows that the distance also is continuous with respect to time when evaluated along the two trajectories. Selecting a positive number $\varepsilon_{d}^{\prime}<\varepsilon_{d}$, as discussed above, there exist $\varepsilon_{T T}^{\prime}\left(\varepsilon_{d}^{\prime}\right)>0$ such that $\left\|e_{T T}(t, j)\right\|<\varepsilon_{T T}^{\prime}$ implies $d(x(t, j), \alpha(t, j))<\varepsilon_{d}^{\prime}$ for all $(t, j) \in \operatorname{dom} x \cap \operatorname{dom} \alpha$. With continuity of $d$ over jumps, we deduce that $d(x(t), \alpha(t))<\varepsilon_{d}^{\prime}$ at the beginning and end of the intervals $I_{\Delta}^{j}$, i.e. for $t=t_{j}$ and for $t=\tau_{j}$. In the intervals $I_{\Delta}^{j}$, the distance $d$ can increase (continuously) however and we now provide a bound for such increase. Select $\varepsilon_{T T}^{\prime \prime} \in\left(0, \varepsilon_{T T}^{\prime}\right)$ such that $C_{d}\left(\sqrt{2} F S \varepsilon_{T T}^{\prime \prime}\right)<\varepsilon_{d}-\varepsilon_{d}^{\prime}$, with $S$ as in the bound $\left|t_{j}-\tau_{j}\right|<S \varepsilon_{T T}^{\prime \prime}$, shown in the proof of Proposition 1, and $C_{d}$ the modulus of continuity of $d$. We now exploit that $d(x(t), \alpha(t))$ equals

$$
\begin{aligned}
& d\left(x\left(t_{j}\right), \alpha\left(t_{j}\right)\right)+d(x(t), \alpha(t))-d\left(x\left(t_{j}\right), \alpha\left(t_{j}\right)\right) \\
\leq \quad & \varepsilon_{d}^{\prime}+C_{d}\left(\left\|\begin{array}{c}
x(t)-x\left(t_{j}\right) \\
\alpha(t)-\alpha\left(t_{j}\right)
\end{array}\right\|\right)
\end{aligned}
$$

and that, by overapproximation of the second term, follows

$$
\begin{aligned}
d(x(t), \alpha(t)) & \leq \varepsilon_{d}^{\prime}+C_{d}\left(\left\|\begin{array}{c}
F\left|t-t_{j}\right| \\
F\left|t-t_{j}\right|
\end{array}\right\|\right) \\
& \leq \varepsilon_{d}^{\prime}+C_{d}\left(\sqrt{2} F S \varepsilon_{T T}^{\prime \prime}\right) .
\end{aligned}
$$

Selecting $\varepsilon_{T T}\left(\varepsilon_{d}\right)<\min \left(\varepsilon_{T T}^{\prime}, \varepsilon_{T T}^{\prime \prime}\right)$, we conclude that (55) holds, and with that condition $\mathrm{A})$, is satisfied.
For proving condition $\mathrm{B}$ ), we note that the set of points $x_{0}$ where $d\left(x_{0}, \alpha_{0}\right)=0$ is the singleton $\left\{x_{0}=\alpha_{0}\right\}$ since, by hypothesis, $\alpha_{0} \notin D \cup g(D)$. With continuity of $d$ and [13, equation (6b)], this implies that indeed for all $\delta_{T T}>0$, there exist $\delta_{d}>0$ such that (56) is satisfied. Combining now conditions A) and B), it follows that if the cl-LTTHS (21)(31) is uniformly asymptotically stable, then, the trajectory $\alpha$ is stable with respect to $d$.

To prove asymptotic stability, we recall that $\alpha$ is an asymptotically stable trajectory of the cl-NTTHS by hypothesis (and Proposition 1) and consequently that, for sufficiently small $\delta_{T T}>0$, solutions $x_{T T}$ to the cl-NTTHS starting from initial conditions $x_{0}$, satisfying $\left\|x_{0}-\alpha_{0}\right\|<\delta_{T T}$, it holds that $\left\|x_{T T}(t)-\alpha(t)\right\| \rightarrow 0$ for $t \rightarrow \infty$. Moreover, as per Theorem 1, we know that $\left|t_{j}-\tau_{j}\right| \rightarrow 0$ for $t \rightarrow \infty$. Again exploiting uniform continuity of $d$ and the upper bound on $d$ given by (57), these convergence aspects allow to conclude asymptotic stability of $\alpha$ with respect to the distance function $d$.

This concludes the proof of Corollary 1.

\section{APPENDIX D}

\section{DERIVATION OF THE LTTHS FROM THE NTTHS}

In this appendix, we show that the LTTHS (21)-(30) is the linearization of the NTTHS (40), (41). The hybrid dynamics of the NTTHS are described in terms of the vector field $f$ of the original NSTHS (2) and the input dependent reset map $g_{T T}^{u}$ given in (41), also graphically represented in Fig. 6. We now perturb the initial condition and input slightly from the reference state-input trajectory $(\alpha(t, j), \mu(t, j))$, that is, we take $x_{T T, \epsilon}\left(t_{0}, 0\right)=\alpha_{0}+\epsilon z_{0}$ and $u_{\epsilon}(t, j)=\mu(t, j)+\epsilon v(t, j)$ for some initial state perturbation $z_{0}$, input perturbation $v(t, j)$ (that may be different for different $j$ ) and scalar perturbation parameter $\epsilon$. Standard results can be used [32] to show that the trajectory of the NTTHS can be expanded in series with respect to $\epsilon$ as $x_{T T, \epsilon}(t, j)=\alpha(t, j)+\epsilon z(t, j)+o(\epsilon)$. It follows that $\dot{x}_{T T, \epsilon}=f(\alpha+\epsilon z+o(\epsilon), \mu+\epsilon v, t, j)$, for $(t, j) \in \operatorname{dom} \alpha$, which can be expanded, in each of the intervals $\left[\tau_{j}, \tau_{j+1}\right]$, as

$$
\begin{aligned}
\dot{\alpha}+\epsilon \dot{z}+o(\epsilon)= & f(\alpha(t, j), \mu(t, j), t, j)+ \\
& \epsilon\left[\mathbf{D}_{1} f(\alpha(t, j), \mu(t, j), t, j) z\right. \\
& \left.+\mathbf{D}_{2} f(\alpha(t, j), \mu(t, j), t, j) v\right]+o(\epsilon) .
\end{aligned}
$$

Matching terms in the expansion allows to conclude that the flow dynamics of the linear approximation about the stateinput trajectory $(\alpha(t, j), \mu(t, j))$ for each continuous time interval $\left[\tau_{j}, \tau_{j+1}\right]$ is given by

$$
\begin{aligned}
\dot{z}= & \mathbf{D}_{1} f(\alpha(t, j), \mu(t, j), t, j) z+ \\
& \mathbf{D}_{2} f(\alpha(t, j), \mu(t, j), t, j) v \\
=: & A(t, j) z+B(t, j) v,
\end{aligned}
$$

that matches the expressions given for $A$ and $B$ in (22) and (23), respectively.

Next, for each jump time $\tau_{j+1}$, we seek a relation between the states $z^{-}\left(\tau_{j+1}\right):=z\left(\tau_{j+1}, j\right)$ and $z^{+}\left(\tau_{j+1}\right):=$ $z\left(\tau_{j+1}, j+1\right)$ that will eventually be equal to (24). Roughly speaking, this corresponds to the linearization of the jump map $g_{T T}^{u}$ in (41). We follow a similar strategy in deriving the linear 
jump map as detailed in [19], but now fitted in the framework of hybrid time.

Consider the $(j+1)$-th event with reference event time $\tau_{j+1}$ and corresponding event time $t_{j+1, \epsilon}$ of the NSTHS. Define $\Delta_{\epsilon}=t_{j+1, \epsilon}-\tau_{j+1}$, which is assumed to be small based on the fact that $x_{T T, \epsilon}$ and $\alpha$ are close to each other and Assumption 3. To make the derivation of (24) concise, below we denote the event time $\tau_{j+1}$ simply by $\tau$. Furthermore, when we refer to $x_{T T, \epsilon}^{-}$, one should read $x_{T T, \epsilon}\left(\tau_{j+1}, j\right)$ and similarly $\alpha^{-}=\alpha\left(\tau_{j+1}, j\right), \mu^{-}=\mu\left(\tau_{j+1}, j\right)$, and $z^{-}=z\left(\tau_{j+1}, j\right)$. Analogously, for the right limits at the time $\tau$, we employ the notation $x_{T T, \epsilon}^{+}=x_{T T, \epsilon}\left(\tau_{j+1}, j+1\right), \alpha^{+}=\alpha\left(\tau_{j+1}, j+1\right)$, $\mu^{+}=\mu\left(\tau_{j+1}, j+1\right)$, and $z^{+}=z\left(\tau_{j+1}, j+1\right)$.

The jump map $g_{T T}^{u}$ in (41) for the event $j+1$ constitutes phases of flow to and from the event time $t_{j+1, \epsilon}$ that depends on $\epsilon$ and the choice of $v(t, j)$. These flows can be captured by extending the solution $z$, using the continuous time part of (21), to form $\bar{z}$ (where states for consecutive counter are related via a 'to be defined" jump map) in a similar fashion as done for the reference trajectory $\alpha$ as explained in Section III-A. This allows us to form the extended trajectory of the NTTHS which, due to the locally Lipschitz property of the vector field $f$, is the same as that of the original NSTHS, i.e. $\bar{x}_{\epsilon}(t, j):=\bar{x}_{T T, \epsilon}(t, j)=\bar{\alpha}(t, j)+\epsilon \bar{z}(t, j)+o(\epsilon)$. It follows that, instead of the jump map $x_{T T, \epsilon}^{+}=g_{T T}^{u}\left(x_{T T, \epsilon}^{-}, \tau, j\right)$, we can consider

$$
\bar{x}_{\epsilon}\left(\tau+\Delta_{\epsilon}, j+1\right)=g\left(\bar{x}_{\epsilon}\left(\tau+\Delta_{\epsilon}, j\right), \tau+\Delta_{\epsilon}, j\right) .
$$

and use a series expansion to account for the difference in time. Again to keep notation concise, we append the states $\bar{\alpha}, \bar{x}_{\epsilon}$, and $\bar{z}$ with a superscript, i.e. $(\cdot)^{\triangleleft}$ to denote that it is the left limit for the event time $t_{j+1, \epsilon}=\tau_{j+1}+\Delta_{\epsilon}$, e.g. $\bar{z}^{\triangleleft}=\bar{z}\left(t_{j+1, \epsilon}, j\right)$. For the right limit we employ the superscript $(\cdot)^{\triangleright}$, e.g. $\bar{z}^{\triangleright}=\bar{z}\left(t_{j+1, \epsilon}, j+1\right)$. For the state $\bar{x}_{\epsilon}^{\triangleleft}$ we find

$$
\begin{aligned}
\bar{x}_{\epsilon}^{\triangleleft} & =\bar{\alpha}^{\triangleleft}+\epsilon \bar{z}^{\triangleleft}+o(\epsilon) \\
& =\alpha^{-}+\dot{\alpha}^{-} \Delta_{0}^{\prime} \epsilon+\left(z^{-}+\dot{z}^{-} \Delta_{0}^{\prime} \epsilon\right) \epsilon+o(\epsilon) \\
& =\alpha^{-}+\left(\dot{\alpha}^{-} \Delta_{0}^{\prime}+z^{-}\right) \epsilon+o(\epsilon)
\end{aligned}
$$

where $\Delta_{0}^{\prime}=\left.\frac{\partial \Delta_{\epsilon}}{\partial \epsilon}\right|_{\epsilon=0}$ and $\dot{\alpha}^{-}=f\left(\alpha^{-}, \mu^{-}, \tau, j\right)$ and similarly that

$$
\begin{aligned}
\bar{x}_{\epsilon}^{\triangleright} & =\bar{\alpha}^{\triangleright}+\epsilon \bar{z}^{\triangleright}+o(\epsilon) \\
& =\alpha^{+}+\left(\dot{\alpha}^{+} \Delta_{0}^{\prime}+z^{+}\right) \epsilon+o(\epsilon)
\end{aligned}
$$

where $\dot{\alpha}^{+}=f\left(\alpha^{+}, \mu^{+}, \tau, j+1\right)$. These expansions make use of the dependence of the jump time difference $\Delta_{\epsilon}$ on $\epsilon$. More precisely, they contain the derivative of $\Delta_{\epsilon}$ with respect $\epsilon$ evaluated at zero. From Assumption 2, it follows that for the reference jump at time $\tau$ holds that $\gamma_{\alpha}\left(\alpha^{-}, \tau, j\right)=0$ since, by definition, the state will be in the jump set $D$. Similarly, the definition of $t_{j+1, \epsilon}$ implies $\gamma_{\alpha}\left(\bar{x}_{\epsilon}\left(t_{j+1, \epsilon}, j\right), t_{j+1, \epsilon}, j\right)=0$, or formulated differently that $\gamma_{\alpha}\left(x_{\epsilon}^{\triangleleft}, \tau+\Delta_{\epsilon}, j\right)=0$. Using (59), this can rewritten as

$$
\begin{aligned}
\gamma_{\alpha}\left(\alpha^{-}, \tau, j\right)+\mathbf{D}_{1} \gamma_{\alpha}\left(\alpha^{-}, \tau, j\right)\left(\dot{\alpha}^{-} \Delta_{0}^{\prime}+z^{-}\right) \epsilon+ & \\
\mathbf{D}_{2} \gamma_{\alpha}\left(\alpha^{-}, \tau, j\right) \Delta_{0}^{\prime} \epsilon+o(\epsilon) & =0 .
\end{aligned}
$$

Since this needs to hold for all $\epsilon$ and since $\gamma_{\alpha}\left(\alpha^{-}, \tau, j\right)=0$, it follows that

$$
\Delta_{0}^{\prime}=-\frac{\mathbf{D}_{1} \gamma_{\alpha}\left(\alpha^{-}, \tau, j\right)}{\mathbf{D}_{1} \gamma_{\alpha}\left(\alpha^{-}, \tau, j\right) \dot{\alpha}^{-}+\mathbf{D}_{2} \gamma_{\alpha}\left(\alpha^{-}, \tau, j\right)} z^{-} .
$$

Note that the transversality assumption (Assumption 3) implies that the denominator in (61) is nonzero.

Incorporating (59) and (60) in (58) and expanding in series with respect to $\epsilon$ gives

$$
\begin{aligned}
\bar{x}_{\epsilon}^{\triangleright}= & \alpha^{+}+\left(\dot{\alpha}^{+} \Delta_{0}^{\prime}+z^{+}\right) \epsilon+o(\epsilon) \\
= & g\left(\alpha^{-}+\left(\dot{\alpha}^{-} \Delta_{0}^{\prime}+z^{-}\right) \epsilon+o(\epsilon), \tau+\Delta_{t}(\epsilon), j\right) \\
= & g\left(\alpha^{-}, \tau, j\right)+\mathbf{D}_{1} g\left(\alpha^{-}, \tau, j\right)\left(\dot{\alpha}^{-} \Delta_{0}^{\prime}+z^{-}\right) \epsilon+ \\
& \mathbf{D}_{2} g\left(\alpha^{-}, \tau, j\right) \Delta_{0}^{\prime} \epsilon+o(\epsilon) .
\end{aligned}
$$

Now we recall that $\alpha^{+}=g\left(\alpha^{-}, \tau, j\right)$ and match terms of $\epsilon$ to obtain $z^{+}=\left(-\dot{\alpha}^{+}+\dot{g}^{-}\right) \Delta_{0}^{\prime}+\mathbf{D}_{1} g\left(\alpha^{-}, \tau, j\right) z^{-}$, where $\dot{g}^{-}=\mathbf{D}_{1} g\left(\alpha^{-}, \tau, j\right) \dot{\alpha}^{-}+\mathbf{D}_{2} g\left(\alpha^{-}, \tau, j\right)$. After incorporating (61), it follows that the linearized jump map satisfies

$$
z^{+}=\left[\left(\dot{\alpha}^{+}-\dot{g}^{-}\right) \frac{\mathbf{D}_{1} \gamma_{\alpha}\left(\alpha^{-}, \tau, j\right)}{\dot{\gamma}_{\alpha}^{-}}+\mathbf{D}_{1} g\left(\alpha^{-}, \tau, j\right)\right] z^{-},
$$

with $\dot{\gamma}_{\alpha}^{-}=\mathbf{D}_{1} \gamma_{\alpha}\left(\alpha^{-}, \tau, j\right) \cdot \dot{\alpha}^{-}+\mathbf{D}_{2} \gamma_{\alpha}\left(\alpha^{-}, \tau, j\right) \cdot 1$, which is equivalent to (24).

\section{REFERENCES}

[1] R. Goebel, R. G. Sanfelice, and A. R. Teel, Hybrid Dynamical Systems. Princeton: Princeton University Press, 2012.

[2] R. I. Leine and H. Nijmeijer, Dynamics and Bifurcations of Non-Smooth Mechanical Systems. Berlin: Springer Verlag, 2004, volume 18 of Lecture Notes in Applied and Computational Mechanics.

[3] B. Brogliato, Nonsmooth Mechanics. Models, Dynamics and Control, 2nd ed. Berlin: Springer, 1999.

[4] V. Acary and B. Brogliato, Numerical Methods for Nonsmooth Dynamical Systems. Springer, 2008.

[5] R. Ronsse, P. Lefévre, and R. Sepulchre, "Sensorless stabilization of bounce juggling," IEEE Transactions on Robotics, vol. 22, no. 1, pp. 147-159, 2006.

[6] B. Morris and J. W. Grizzle, "Hybrid invariant manifolds in systems with impulsive effects with application to periodic locomotion in bipedal robots," IEEE Transactions on Automatic Control, vol. 54, no. 8, pp. 1751-1764, 2009.

[7] J. Reher, E. A. Cousineau, A. Hereid, C. M. Hubicki, and A. D. Ames, "Realizing dynamic and efficient bipedal locomotion on the humanoid robot durus," in 2016 IEEE International Conference on Robotics and Automation (ICRA), 2016, pp. 1794-1801.

[8] B. Brogliato, S. Niculescu, and M. Monteiro-Marques, "On tracking control of a class of complementary-slackness hybrid mechanical systems," Systems \& Control Letters, vol. 39, no. 4, pp. 255-266, 2000.

[9] R. G. Sanfelice, J. J. B. Biemond, N. van de Wouw, and W. P. M. H. Heemels, "An embedding approach for the design of state-feedback tracking controllers for references with jumps," International Journal of Robust and Nonlinear Control, vol. 24, no. 11, pp. 869-874, 2014.

[10] R. I. Leine and N. van de Wouw, "Uniform convergence of monotone measure differential inclusions: With application to the control of mechanical systems with unilateral constraints," International Journal of Bifurcation and Chaos, vol. 18, no. 5, pp. 1435-1457, 2008.

[11] L. Menini and A. Tornambè, "Asymptotic tracking of periodic trajectories for a simple mechanical system subject to nonsmooth impacts," IEEE Transactions on Automatic Control, vol. 46, no. 7, pp. 1122-1126, 2001.

[12] R. I. Leine and N. van de Wouw, Stability and Convergence of Mechanical Systems with Unilateral Constraints. Berlin: Springer Verlag, 2008, volume 36 of Lecture Notes in Applied and Computational Mechanics.

[13] J. J. B. Biemond, N. van de Wouw, W. P. M. H. Heemels, and H. Nijmeijer, "Tracking control for hybrid systems with state-triggered jumps," IEEE Transactions on Automatic Control, vol. 58, no. 4, pp. 876-890, 2013. 
[14] S. Galeani, L. Menini, and A. Potini, "Robust trajectory tracking for a class of hybrid systems: an internal model principle approach," IEEE Transactions on Automatic Control, vol. 57, no. 2, pp. 344-359, 2012.

[15] F. Forni, A. R. Teel, and L. Zaccarian, "Follow the bouncing ball: global results on tracking and state estimation with impacts," IEEE Transactions on Automatic Control, vol. 58, no. 6, pp. 1470-1485, 2013.

[16] J. J. B. Biemond, W. P. M. H. Heemels, R. G. Sanfelice, and N. van de Wouw, "Distance function design and lyapunov techniques for the stability of hybrid trajectories," Automatica, vol. 73, pp. 38-46, 2016.

[17] T. Yang, F. Wu, and L. Zhang, "Tracking control of hybrid systems with state-triggered jumps and stochastic events and its application," IET Control Theory Appl., vol. 11, no. 7, pp. 1024-1033, 2017.

[18] J. Kim, H. Shim, and J. H. Seo, "Tracking control for hybrid systems with state jumps using gluing function," in Proceedings of the 55th IEEE Conference on Decision and Control, Las Vegas, USA, December 2016 , pp. 3006-3011.

[19] A. Saccon, N. van de Wouw, and H. Nijmeijer, "Sensitivity analysis of hybrid systems with state jumps with application to trajectory tracking," in Proceedings of the 53rd IEEE Conference on Decision and Control, Los Angeles, USA, December 2014, pp. 3065-3070.

[20] M. W. L. M. Rijnen, A. Saccon, and H. Nijmeijer, "On optimal trajectory tracking for mechanical systems with unilateral constraints," in Proceedings of the 54th IEEE Conference on Decision and Control, Osaka, Japan, December 2015, pp. 2561-2566.

[21] M. W. L. M. Rijnen, E. B. C. de Mooij, S. Traversaro, F. Nori, N. van de Wouw, A. Saccon, and H. Nijmeijer, "Control of humanoid robot motions with impacts: Numerical experiments with reference spreading control," in Proceedings of the IEEE International Conference on Robotics and Automation, Singapore, May 2017, pp. 4102-4107.

[22] M. E. Broucke and A. Arapostathis, "Continuous selections of trajectories of hybrid systems," Systems \& Control Letters, vol. 47, no. 2, pp. 149-157, 2002.

[23] S. Galeani, L. Menini, and A. Potini, "Trajectory tracking in linear hybrid systems: an internal model principle approach," in Proceedings of the 2008 American Control Conference, Seattle, USA, June 2008, pp. $4627-4632$.

[24] F. Forni, A. R. Teel, and L. Zaccarian, "Tracking control in billiards using mirrors without smoke, part i: Lyapunov-based local tracking in polyhedral regions," in Proceedings of the 50th IEEE Conference on Decision and Control, Orlando, USA, December 2011, pp. 3283-3288.

[25] _ _ "Tracking control in billiards using mirrors without smoke, part ii: additional Lyapunov-based local and global results," in Proceedings of the 50th IEEE Conference on Decision and Control, Orlando, USA, December 2011, pp. 3289-3294.

[26] W. Michiels and D. Roose, "Sensitivity to perturbations in variable structure systems," Journal of Computational and Applied Mathematics, vol. 132, no. 1, pp. 127-140, 2001.

[27] A. N. Michel, L. Hou, and D. Liu, Stability of Dynamical Systems. Boston: Birkhäuser Boston, 2008.

[28] H. Ye, A. N. Michel, and L. Hou, "Stability analysis for systems with impulse effects," IEEE Transactions on Automatic Control, vol. 43, no. 12 , pp. $1719-1723,1998$.

[29] M. di Bernardo, C. J. Budd, A. R. Champneys, and P. Kowalczyk, Piecewise-smooth Dynamical Systems: Theory and Applications. London, UK: Springer Verlag, 2008.

[30] J. K. Hale, Ordinary Differential Equations, 2nd ed. Florida: Krieger Publishing Company, 1980

[31] H. Ye, A. N. Michel, and L. Hou, "Stability analysis for hybrid dynamical systems," IEEE Transactions on Automatic Control, vol. 43, no. 4, pp. 461-474, 1998.

[32] H. K. Khalil, Nonlinear Systems, 2nd ed. Upper Saddle River: Prentice Hall, 2002.

Mark Rijnen obtained his Master's degree (cum laude) in Mechanical Engineering from the Eindhoven University of Technology, Eindhoven, The Netherlands in 2014. He is currently pursuing the Ph.D. degree at the Department of Mechanical Engineering, Eindhoven University of Technology. His research interests include modeling, analysis and control of mechanical systems, and nonsmooth and nonlinear systems.
Benjamin Biemond obtained his Master's degree in Mechanical Engineering in 2009. Subsequently, he started a $\mathrm{PhD}$ at the Eindhoven University of Technology, under the supervision of prof.dr. Henk Nijmeijer and dr.ir. Nathan van de Wouw. Benjamin received his PhD degree in March 2013 with his thesis "Nonsmooth dynamical systems: On stability of hybrid trajectories and bifurcations of discontinuous systems". Subsequently, Benjamin started as a postdoc with prof.dr. Wim Michiels at the Numerical Analysis and Applied Mathematics Section of the Computer Science Department of KU Leuven. He studied the dynamics and control of nonlinear systems with time-delays. $\mathrm{He}$ provided new results at the intersection of the domains of nonsmooth systems and time-delay systems. He received a FWO Pegasus Marie Curie Fellowship. In September 2016, Benjamin has started as Dynamics \& Control Specialist at the Department of Optomechatronics at the Netherlands Organization for Applied Scientific Research TNO, Delft.

Nathan van de Wouw (born, 1970) obtained his M.Sc.-degree (with honours) and Ph.D.-degree in Mechanical Engineering from the Eindhoven University of Technology, the Netherlands, in 1994 and 1999, respectively. He currently holds a full professor position at the Mechanical Engineering Department of the Eindhoven University of Technology, the Netherlands. Nathan van de Wouw also holds an adjunct full professor position at the University of Minnesota, U.S.A and a (part-time) full professor position at the Delft University of Technology, the Netherlands. He has been working at Philips Applied Technologies, The Netherlands, in 2000 and at the Netherlands Organisation for Applied Scientific Research, The Netherlands, in 2001. He has been a visiting professor at the University of California Santa Barbara, U.S.A., in 2006/2007, at the University of Melbourne, Australia, in 2009/2010 and at the University of Minnesota, U.S.A., in 2012 and 2013. He has published a large number of journal and conference papers and the books 'Uniform Output Regulation of Nonlinear Systems: A convergent Dynamics Approach' with A.V. Pavlov and H. Nijmeijer (Birkhauser, 2005) and 'Stability and Convergence of Mechanical Systems with Unilateral Constraints' with R.I. Leine (Springer-Verlag, 2008). In 2015, he received the IEEE Control Systems Technology Award "For the development and application of of variable-gain control techniques for high-performance motion systems".

Alessandro Saccon received the laurea degree cum laude in computer engineering and the $\mathrm{Ph} . \mathrm{D}$. degree in control system theory from the University of Padova, Italy, in 2002 and 2006. His thesis received the Claudio Maffezzoni best $\mathrm{PhD}$ thesis award by the Politecnico di Milano. After the completion of his PhD studies, he held until 2009 a research and development position at University of Padova in joint collaboration with Ducati Corse. From 2009 until 2012, he held a post-doctoral research position at the Instituto Superior Técnico, Lisbon, Portugal, sponsored by the Portuguese Science and Technology Foundation (FCT). Since 2013, he is an Assistant Professor on nonlinear control and robotics at the Department of Mechanical Engineering, Eindhoven University of Technology, the Netherlands. His areas of expertise include optimal constrained motion planning and control of mechanical systems subject to unilateral constraints.

Henk Nijmeijer (1955) is a full professor at Eindhoven, and chairs the Dynamics and Control group. He has published a large number of journal and conference papers, and several books, and is or was at the editorial board of numerous journals. He is an editor of Communications in Nonlinear Science and Numerical Simulations. He is a fellow of the IEEE since 2000 and was awarded in 1990 the IEE Heaviside premium. He is appointed honorary knight of the 'golden feedback loop' (NTNU) in 2011. He is since 2011 an IFAC Council Member. Per January 2015 he is scientific director of the Dutch Institute of Systems and Control (DISC). He is recipient of the 2015 IEEE Control Systems Technology Award. 